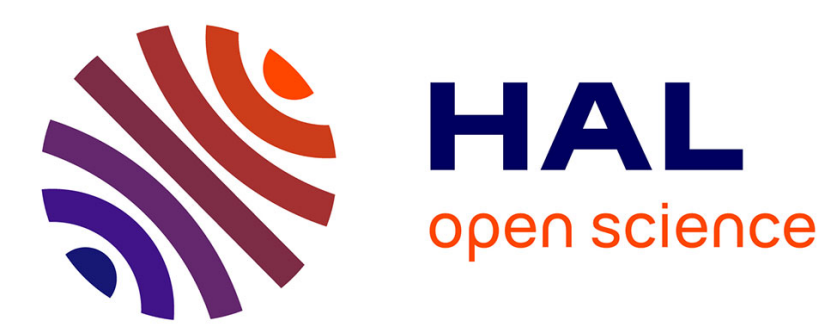

\title{
Natural decomposition of processes and weak Dirichlet processes
}

François Coquet, Adam Jakubowski, Jean Mémin, Leszek Slominski

\section{To cite this version:}

François Coquet, Adam Jakubowski, Jean Mémin, Leszek Slominski. Natural decomposition of processes and weak Dirichlet processes. Lecture Notes in Mathematics, 2006, 1874, pp.81-116. hal00001360v2

\section{HAL Id: hal-00001360 \\ https://hal.science/hal-00001360v2}

Submitted on 5 Apr 2004

HAL is a multi-disciplinary open access archive for the deposit and dissemination of scientific research documents, whether they are published or not. The documents may come from teaching and research institutions in France or abroad, or from public or private research centers.
L'archive ouverte pluridisciplinaire HAL, est destinée au dépôt et à la diffusion de documents scientifiques de niveau recherche, publiés ou non, émanant des établissements d'enseignement et de recherche français ou étrangers, des laboratoires publics ou privés. 


\title{
Natural decomposition of processes and weak Dirichlet processes
}

\author{
François Coquet*, Adam Jakubowski**,1 \\ Jean Mémin ${ }^{* * *}$ and Leszek Słomiński ${ }^{* *, 2}$ \\ ${ }^{*}$ LMAH, Université du Havre \\ 25 rue Philippe Lebon, 76063 Le Havre Cedex, France \\ ** Faculty of Mathematics and Computer Science, \\ N. Copernicus University, ul. Chopina, 87-100 Toruń, Poland \\ *** IRMAR, Université de Rennes 1 \\ Campus de Beaulieu, 35042 Rennes Cedex, France
}

\begin{abstract}
A class of stochastic processes, called "weak Dirichlet processes", is introduced and its properties are investigated in detail. This class is much larger than the class of Dirichlet processes. It is closed under $C^{1}$-transformations and under absolutely continuous change of measure. If a weak Dirichlet process has finite energy, as defined by Graversen and Rao, its Doob-Meyer type decomposition is unique. The developed methods have been applied to a study of generalized martingale convolutions.
\end{abstract}

Mathematics Subject Classification: 60G48, 60H05

Keywords: weak Dirichlet processes, Dirichlet processes, semimartingales, finite energy processes, quadratic variation, mutual quadratic covariation, Ito's formula, generalized martingale convolution.

\footnotetext{
${ }^{1}$ Supported in part by Komitet Badań Naukowych under Grant No 1 P03A 02226 and completed while the author was visiting Université de Rennes I.

${ }^{2}$ Supported in part by Komitet Badań Naukowych under Grant No 1 P03A 02226 and completed while the author was visiting Université de Rennes I.
} 


\section{Introduction}

The quadratic variation of a stochastic process as well as the mutual covariation of two stochastic processes have been well-known for a long time to be at the core of the theory of stochastic integration, was it only because the quadratic variation appears explicitely in Ito's formula for semimartingales. And indeed, every attempt to generalize Ito's calculus to a wider class of integrators (for instance Dirichlet processes) or to functions less regular than $\mathcal{C}^{2}$ functions has to deal with quadratic variations or covariations of the processes that appear. In this aspect, the most enlightening work is perhaps Föllmer's paper ([7]).

On the other hand, it was proven by Graversen and Rao ([9]) that the existence of a Doob-Meyer type decompositon for a process $X$ is narrowly linked to the fact that $X$ has a finite energy, which is a somewhat weaker assumption than the existence of a quadratic variation for $X$. The most well-known class of processes with finite energy (beyond the class of semimartingales) is the class of Dirichlet processes. A larger class has been recently introduced by Errami and Russo ([5]) under the name "weak Dirichlet processes". The present paper explores some desirable properties of such processes. Although our definition of a quadratic variation is different from Errami and Russo's one -it is in a way more classical- at any rate both coincide as far as semimartingales are concerned. The other noticeable difference is that throughout the paper we deal with non continuous processes.

In part 2., we give an as explicit as possible link between quadratic variation, energy, Dirichlet processes, weak Dirichlet processes, and "natural" (that is, "Doob-Meyer type") decomposition.

Part 3. is devoted to prove that any $\mathcal{C}^{1}$ function of a weak Dirichlet process is again a weak Dirichlet process. We are able to give an explicit Ito-type formula for $\mathcal{C}^{2}$ transformations, but we could only find an explicit formula for the martingale part in the general case.

Part 4. which is closest to Errami and Russo's work mentionned above, deals with processes $X$ that may be written $X_{t}=\int_{0}^{t} G(t, s) d L_{s}$ where $L$ is a quasileft continuous -but not necessarily continuous- square-integrable martingale and $G$ is a deterministic function. We give two sets of hypotheses under which $X$ is a weak Dirichlet process, and also give its natural decomposition. This section is illustrated through 3 examples, last one giving additionnaly a formula of Fubini type.

At last, we joined as an appendix some counter-examples related to 
quadratic variation or to regularity of paths of processes. Although such examples may be well-known, we could not find any in the litterature, and we hope that they can enlight some of the technical problems we are confronted with here and there in the paper.

\section{Basic notations and results about processes with finite energy and weak Dirichlet processes}

In what follows, we are given a probability space $(\Omega, \mathcal{G}, \mathbf{P})$.

We also fix a positive real number $T$. Unless otherwise stated, every process or filtration will be indexed by $t \in[0, T]$. A filtration $\left(\mathcal{F}_{t}\right)_{t \leq T}$ is denoted by $\mathcal{F}$. All filtrations are assumed to be right-continuous and defined on $(\Omega, \mathcal{G}, \mathbf{P})$ with $\mathcal{F}_{T} \subset \mathcal{G}$.

We are also given a refining sequence $D_{n}$ of subdivisions of $[0, T]$ whose mesh goes to 0 when $n \rightarrow \infty$. For every $n, D_{n}=\left\{0=t_{0}^{n}, t_{1}^{n}, \ldots, t_{N(n)}^{n}=T\right\}$.

We work with processes with a.s. right-continuous trajectories with left limits (such a process is called càdlàg), null in 0 and, unless otherwise stated, admitting a finite energy in the sense defined below following Graversen and Rao ([9]):

Definition 2.1 We say that $X$ is a process of finite energy if

$$
\sup _{n} E\left[\sum_{t_{i}^{n} \in D_{n}}\left(X_{t_{i}^{n}}-X_{t_{i-1}^{n}}\right)^{2}\right]<+\infty .
$$

This "sup" will be denoted $\mathcal{E} n(X)$.

Of course, if $X$ has a finite energy, $\left|X_{t}\right|^{2}$ is integrable for every $t \leq T$ and also $\sum_{s \leq T} \Delta X_{s}^{2}$ is integrable.

We recall Graversen and Rao's main result in [9]:

Theorem 2.1 If $X$ is a process with finite energy, then we can write $X$ as a sum $X=M+A$, where $M$ is a square-integrable martingale and $A$ is a predictable process such that there exists a subsequence $\left(D_{n_{j}}\right)$ of $\left(D_{n}\right)$ satisfying

$$
E\left[\sum_{t_{i}^{n_{j}} \in D_{n_{j}}, t_{i}^{n_{j}} \leq t}\left(A_{t_{i}^{n_{j}}}-A_{t_{i-1}}\right)\left(N_{t_{i}^{n_{j}}}-N_{t_{i-1}^{n_{j}}}\right)\right] \longrightarrow 0
$$


as $j \rightarrow \infty$ for all square integrable martingale $N$.

If, moreover, $X=M^{\prime}+A^{\prime}$ is any other such decomposition, the process $A-A^{\prime}$ is a continuous martingale.

At last, if we write

$$
M_{t}^{n}=\sum_{t_{i}^{n} \in D_{n}, t_{i}^{n} \leq t}\left[X_{t_{i}^{n}}-E\left[X_{t_{i}^{n}} / \mathcal{F}_{t_{i-1}^{n}}\right]\right],
$$

then for all $t \in \bigcup_{n} D_{n}, M_{t}$ is the weak limit in $\sigma\left(\mathbf{L}^{2}, \mathbf{L}^{2}\right)$ of the sequence, $\left(M_{t}^{n_{j}}\right)$.

Such a decomposition of $X$ is a Doob-Meyer type decomposition: the predictable process $A$ with the property of convergence (2) is a "natural" process. In this section we will discuss the case when the decomposition $X=M+A$ in Theorem 2.1 is unique. Such Doob-Meyer decomposition will be called the natural decomposition of $X$.

We will use the notion of weak Dirichlet process introduced by Errami and Russo ([5]) in a slighty different context.

Definition 2.2 We say that $X$ is a weak Dirichlet process if it admits a decomposition $X=M+A$, where $M$ is a local martingale and $A$ is a predictable process such as $[A, N]=0$ for all continuous local martingale $N$.

In the above definition and in the sequel we use the notion of mutual covariation and quadratic variation in the following sense taken from [2].

Definition 2.3 (i) Processes $X$ and $Y$ admit a quadratic (mutual) covariation along $\left(D_{n}\right)$ if there exists a càdlàg process denoted $[X, Y]$ with for every $t \leq T$

$$
[X, Y]_{t}=[X, Y]_{t}^{c}+\sum_{s \leq t} \Delta X_{s} \Delta Y_{s}
$$

and

$$
S^{n}(X, Y)_{t}:=\sum_{t_{i}^{n} \in D_{n}, t_{i}^{n} \leq t}\left(X_{t_{i+1}^{n}}-X_{t_{i}^{n}}\right)\left(Y_{t_{i+1}^{n}}-Y_{t_{i}^{n}}\right) \stackrel{\mathbf{P}}{\longrightarrow}[X, Y]_{t} \text { as } n \rightarrow \infty .
$$

(ii) The process $X$ admits a quadratic variation along $\left(D_{n}\right)$ if there exists a càdlàg process denoted $[X, X]$ with for every $t \leq T$

$$
[X, X]_{t}=[X, X]_{t}^{c}+\sum_{s \leq t} \Delta X_{s}^{2}
$$


and

$$
S^{n}(X, X)_{t}:=\sum_{t_{i}^{n} \in D_{n}, t_{i}^{n} \leq t}\left(X_{t_{i+1}^{n}}-X_{t_{i}^{n}}\right)^{2} \stackrel{\mathbf{P}}{\longrightarrow}[X, X]_{t} \text { as } n \rightarrow \infty
$$

Remark 2.1 (i) The decomposition $X=M+A$ of a weak Dirichlet process is unique.

To see this suppose that we have decompositions $X=M+A=M^{\prime}+A^{\prime}$ with $A$ and $A^{\prime}$ predictable and verifying $[A, N]=\left[A, N^{\prime}\right]=0$ for every continuous local martingale $N$. Then $A-A^{\prime}$ is a predictable local martingale, hence a continuous local martingale. Then

$\left[A-A^{\prime}\right]_{T}=\left[A-A^{\prime}, A\right]_{T}-\left[A-A^{\prime}, A^{\prime}\right]_{T}=0$

and we deduce that $A=A^{\prime}$.

(ii) A weak Dirichlet process $X$ need not admit a quadratic variation. We know only that for every continuous martingale $N$ there exists the covariation $[X, N]$.

(iii) Of course, in general, a decomposition $X=M+A$ with a martingale $M$ and a predictable process $A$, does not imply that $[A, N]=0$ for every continuous martingale $N$, when $[A, N]$ exists. For example, take $A$ as a continuous martingale and $N=A$.

The class of weak Dirichlet processses is much larger than the class of Dirichlet processes. We recall:

Definition 2.4 A Dirichlet process is the sum of a local martingale and a continuous process whose quadratic variation is identically zero.

Remark 2.2 Note that a Dirichlet process admits a quadratic variation, which is equal to the quadratic variation of its martingale part. Our definition of a quadratic variation, which follows Föllmer's one in [7] is weaker than the definition in [8], and slightly different from Russo and Vallois' one in [14]. However the three of them coincide as far as semimartingales are concerned, and a Dirichlet process according to the definition in [8] is also Dirichlet according to the two other ones.

The following notion of pre-quadratic variation is weaker than the quadratic variation one; however the two notions coincide under stronger assumptions, as will be seen below. 
Definition 2.5 A process $X$ (not necessarily càdlàg) admits a pre-quadratic variation along $\left(D_{n}\right)$ if there exists an increasing process denoted $S(X, X)$ with for every $t \leq T$

$$
S^{n}(X, X)_{t}:=\sum_{t_{i}^{n} \in D_{n}, t_{i}^{n} \leq t}\left(X_{t_{i+1}^{n}}-X_{t_{i}^{n}}\right)^{2} \stackrel{\mathbf{P}}{\longrightarrow} S(X, X)_{t} \quad \text { as } n \rightarrow \infty .
$$

Remark 2.3 We can find examples of continuous processes $X$ such that $S(X, X)$ is defined but not continuous (see example in Annex), hence $X$ does not admit a quadratic variation.

For every $t \leq T$ denoting $\pi_{t}$ any subdivision of $[0, t]$, we consider the sum

$$
S^{\pi_{t}}(X, X):=\sum_{t_{i} \in \pi_{t}, i>0}\left(X_{t_{i}}-X_{t_{i-1}}\right)^{2}
$$

Proposition 2.1 If a càdlàg process $X$ admits a pre-quadratic variation $S$ with the following property $(S)$ :

$S(X, X)$ is right continuous and for every $t \leq T, S^{\pi_{t}}(X, X) \stackrel{\mathrm{P}}{\longrightarrow} S(X, X)_{t}$ as the mesh of $\pi_{t}$ tends to 0.

Then, $S(X, X)$ is the quadratic variation of $X$ along any sequence $\left(D_{n}\right)$ of subdivisions of $[0, T]$, whose mesh tends to 0 .

This result is proved in ([10]), Lemme (3.11).

Remark 2.4 (i) The class of Dirichlet processes is larger than the space $\mathbf{H}^{2}$ of semimartingales. Every continuous function admitting a quadratic variation equal to zero is a deterministic Dirichlet process.

(ii) Every continuous function is a deterministic weak Dirichlet process: Actually, let us consider a bounded continuous martingale $N$ nul in 0 , we have

$E\left(\left|\sum_{t_{i}^{n} \in D_{n}}\left(f\left(t_{i+1}^{n}\right)-f\left(t_{i}^{n}\right)\right)\left(N_{t_{i+1}^{n}}-N_{t_{i}^{n}}\right)\right|^{2}\right) \leq \sup _{t_{i}^{n}}\left(f\left(t_{i+1}^{n}\right)-f\left(t_{i}^{n}\right)\right)^{2} E\left(N_{T}\right)^{2}$.

and, from continuity of $f$ this last term tends to 0 when $n \rightarrow \infty$.

We give in Section 4 nondeterministic examples of weak Dirichlet processes, which are not ordinary Dirichlet processes. 
Remark 2.5 The family of processes with finite energy is clearly stable under addition, however we do not know if this stability holds for the family of processes admitting a quadratic variation. Of course this is true for the family of Dirichlet processes.

Theorem 2.2 Assume $X$ is a process with finite energy. The following three conditions are equivalent:

(i) $X$ is a weak Dirichlet process,

(ii) for every continuous local martingale $N$, the quadratic covariation $[X, N]$ is well-defined,

(iii) for every locally square integrable martingale $N$, the quadratic covariation $[X, N]$ is well-defined.

In this case the decomposition $X=M+A$ is unique and it is the natural decomposition expressed in Theorem 2.1.

Proof: $(i) \Rightarrow($ iii $)$ Let us write $X=M+A$ as in Definition 2.2 and consider the decomposition $N=N^{c}+N^{d}$, where $N^{c}$ is the continuous and $N^{d}$ purely discontinuous part of $N$. By the definition of a weak Dirichlet process the covariation $\left[X, N^{c}\right]$ is well-defined. For proving the existence of $\left[X, N^{d}\right]$ we use the following lemma.

Lemma 2.1 Assume that $X$ has a finite energy, and that $N$ is a locally square integrable martingale which is the compensated sum of its jumps. Then $X$ and $N$ admit a covariation such that

$$
[X, N]_{t}=\sum_{s \leq t} \Delta X_{s} \Delta N_{s}
$$

Proof of Lemma 2.1: By using a localizing sequence of stopping times, one can assume that $N$ is a square integrable martingale. One can find a sequence $\left(N^{p}\right)_{p}$ of martingales with finite variation and only a finite number of jumps, such that $N^{p} \rightarrow N$ in $\mathbf{H}^{\mathbf{2}}$.

We have then, for fixed $p$,

$$
\sum_{t_{i}^{n} \in D_{n}, t_{i}^{n} \leq t}\left(X_{t_{i+1}^{n}}-X_{t_{i}^{n}}\right)\left(N_{t_{i+1}^{n}}^{p}-N_{t_{i}^{n}}^{p}\right) \stackrel{\mathbf{P}}{\longrightarrow} \sum_{s \leq t} \Delta X_{s} \Delta N_{s}^{p} .
$$

as $n \rightarrow \infty$. 
On the other hand,

$$
\begin{gathered}
E\left|\sum_{t_{i}^{n} \in D_{n}, t_{i}^{n} \leq t}\left(X_{t_{i+1}^{n}}-X_{t_{i}^{n}}\right)\left(N_{t_{i+1}^{n}}^{p}-N_{t_{i}^{n}}^{p}\right)-\sum_{t_{i}^{n} \in D_{n}, t_{i}^{n} \leq t}\left(X_{t_{i+1}^{n}}-X_{t_{i}^{n}}\right)\left(N_{t_{i+1}^{n}}-N_{t_{i}^{n}}\right)\right| \\
\leq E \mid\left(\sum_{t_{i}^{n} \in D_{n}, t_{i}^{n} \leq t}\left(X_{t_{i+1}^{n}}-X_{t_{i}^{n}}\right)^{2}\right)^{1 / 2} \\
\quad \times\left(\sum_{t_{i}^{n} \in D_{n}, t_{i}^{n} \leq t}\left(\left(N_{t_{i+1}^{n}}-N_{t_{i+1}^{n}}^{p}\right)-\left(N_{t_{i}^{n}}-N_{t_{i}^{n}}^{p}\right)\right)^{2}\right)^{1 / 2} \mid \\
\leq(\mathcal{E} n(X))^{1 / 2} E\left(\left[N-N^{p}, N-N^{p}\right]_{t}\right)^{1 / 2}
\end{gathered}
$$

which goes to 0 as $p \rightarrow \infty$ since $\left[N-N^{p}, N-N^{p}\right]_{t} \stackrel{\mathbf{P}}{\longrightarrow} 0$.

At last,

$$
\begin{gathered}
E\left|\sum_{s \leq t} \Delta X_{s}\left(\Delta N_{s}-\Delta N_{s}^{p}\right)\right| \leq E\left(\left(\sum_{s \leq t} \Delta X_{s}^{2}\right)^{1 / 2}\left(\sum_{s \leq t}\left(\Delta N_{s}-\Delta N_{s}^{p}\right)^{2}\right)^{1 / 2}\right. \\
\leq(\mathcal{E} n(X))^{1 / 2} E\left[\left[N-N^{p}, N-N^{p}\right]_{t}\right]^{1 / 2}
\end{gathered}
$$

which goes to zero as $p$ goes to infinity, hence $\sum_{s \leq t} \Delta X_{s} \Delta N_{s}^{p}$ converges in $L^{1}$ to $\sum_{s \leq t} \Delta X_{s} \Delta N_{s}$.

These three convergences give the lemma.

$($ iii $) \Rightarrow($ ii $)$ is obvious.

(ii) $\Rightarrow(i)$ Let $X=M+A$ be a decomposition from Theorem 2.1 and let $N$ be a continuous local martingale. Define $T_{p}=\inf \left\{t:\left|N_{t}\right| \geq p\right\}$ then $\left(T_{p}\right)$ is a localizing sequence of stopping times. We will prove that for every $p,\left[A, N^{T_{p}}\right]=0$, which implies that also $[A, N]=0$.

By hypothesis we have the convergence $S^{n}\left(X, N^{T_{p}}\right)_{t} \stackrel{\mathrm{P}}{\longrightarrow}\left[X, N^{T_{p}}\right]_{t}$.

Hence we deduce that also $S^{n}\left(A, N^{T_{p}}\right)_{t} \stackrel{\mathrm{P}}{\longrightarrow}\left[A, N^{T_{p}}\right]_{t}$. We will show that in fact

$$
S^{n}\left(A, N^{T_{p}}\right)_{T} \rightarrow\left[A, N^{T_{p}}\right]_{T} \text { in } L^{1} .
$$

To see this, it is sufficient to check uniform integrability of the sequence $\left\{S^{n}\left(A, N^{T_{p}}\right)_{T}\right\}$. Writing $\left|S^{n}\left(X, N^{T_{p}}\right)_{T}\right| \leq S^{n}(X, X)_{T}^{1 / 2} S^{n}\left(N^{T_{p}}, N^{T_{p}}\right)_{T}^{1 / 2}$, and using Hölder inequality, we get:

$$
E\left|S^{n}\left(X, N^{T_{p}}\right)_{T}\right|^{4 / 3} \leq E\left[S^{n}(X, X)_{T}\right] E\left[S^{n}\left(N^{T_{p}}, N^{T_{p}}\right)_{T}^{2}\right] .
$$


Similarly we prove that also $E\left|S^{n}\left(M, N^{T_{p}}\right)_{T}\right|^{4 / 3}<+\infty$.

As a consequence, we deduce uniform integrability of $\left\{\left(S^{n}\left(A, N^{T_{p}}\right)_{T}\right\}\right.$ and (9) holds true. Therefore, in particular

$$
E\left[S^{n}\left(A, N^{T_{p}}\right)_{T}\right] \rightarrow E\left[A, N^{T_{p}}\right]_{T}
$$

and due to $(2)$

$$
E\left[A, N^{T_{p}}\right]_{T}=0 .
$$

Note that the process $\left[A, N^{T_{p}}\right]$ has a finite variation ; moreover, since $N$ is continuous, $\left[A, N^{T_{p}}\right]$ is also a continuous process. Therefore, to get $\left[A, N^{T_{p}}\right]=0$ it is sufficient to prove that $\left[A, N^{T_{p}}\right]$ is a local martingale

Let us consider a bounded stopping time $\tau \leq T$, the same arguments as above give the convergence:

$$
E\left[S^{n}\left(A, N^{T_{p} \wedge \tau}\right)_{T}\right] \rightarrow E\left[A, N^{T_{p} \wedge \tau}\right]_{T}
$$

and $(2)$ gives

$$
E\left[A, N^{T_{p}}\right]_{\tau}=E\left[A, N^{T_{p} \wedge \tau}\right]_{T}=0 .
$$

Hence, it follows easily that the stopped process $[A, N]^{T_{p}}$ is a martingale and $\left[A, N^{T_{p}}\right]=0$. Since $P\left(T_{p}=T\right) \uparrow 1$, the proof of the last implication is completed.

Finally, note that the uniqueness of the decomposition in Theorem 2.1 is an easy consequence of the fact that $X$ is a weak Dirichlet process.

We get immediately the following

Corollary 2.1 Let us consider a weak Dirichlet process $X$

(i) If $Q$ is a probability measure absolutely continuous with respect to $\mathbf{P}$, then $X$ is a $Q$ weak Dirichlet process.

(ii) For an $a>0$ we define $\hat{X}=\sum_{s \leq .} \Delta X_{s} 1_{\Delta\left|X_{s}\right|>a}$, then $X-\hat{X}$ is a weak Dirichlet process.

Now, we will consider processes with finite energy $X$ admitting additionally a quadratic variation $[X, X]$. Then of course $E[X, X]_{T}<\infty$.

Theorem 2.3 Assume $X$ is a weak Dirichlet process with finite energy, admitting a quadratic variation process.

(i) In the natural decomposition $X=M+A, M$ is a square integrable martingale and $A$ has an integrable quadratic variation. 
(ii) The natural decomposition is minimal in the following sense. If $X=$ $M^{\prime}+A^{\prime}$ is another decomposition with a local martingale $M^{\prime}$ and a predictable process $A^{\prime}$

then $\left[A^{\prime} A^{\prime}\right]$ is well defined and:

$$
\left[A^{\prime}, A^{\prime}\right]=\left[M-M^{\prime}, M-M^{\prime}\right]+[A, A] .
$$

Proof: (i) To begin with, we notice that

$$
E\left[\sum_{s \leq T} \Delta A_{s}^{2}\right]<\infty
$$

actually, for every predictable stopping time $S, \Delta A_{S}=E\left[\Delta X_{S} \mid \mathcal{F}_{S^{-}}\right]$, hence $E\left[\sum_{s \leq T} \Delta A_{s}^{2}\right] \leq E\left[\sum_{s \leq T} \Delta X_{s}^{2}\right]<\infty$. It follows that $E\left[\sum_{s \leq T} \Delta M_{s}^{2}\right]<\infty$ and $M$ is a locally square integrable martingale.

Let us consider the decomposition $M=M^{c}+M^{d}$ where $M^{c}$ is the continuous part of $M$ and $M^{d}$ its purely discontinuous part. Writing $\left[M^{d}, A\right]=$ $\left[M^{d}, X\right]-\left[M^{d}, M^{d}\right]$, we get the existence of $\left[M^{d}, A\right]$. Using the property of decomposition $X=M+A$, we have $[M, A]=\left[M^{d}, A\right]$. From Lemma 2.1 we deduce:

$$
[M, A]=\sum_{s \leq \cdot} \Delta M_{s} \Delta X_{s}-\sum_{s \leq \cdot} \Delta M_{s}^{2}=\sum_{s \leq \cdot} \Delta M_{s} \Delta A_{s} .
$$

Now, by the definition of quadratic variation of $X$ and $M$ one gets the existence of $[A, A]$ :

$$
[A, A]=[X, X]-2[M, A]-[M, M]
$$

Finally,

$$
E\left([M, M]_{T}+[A, A]_{T}\right) \leq E[X, X]_{T}+2 E\left[\sum_{s \leq T} \Delta M_{s}^{2}\right]^{1 / 2} E\left[\sum_{s \leq T} \Delta A_{s}^{2}\right]^{1 / 2}<\infty .
$$

(ii) Since $A^{\prime}=M+A-M^{\prime}$, by linearity $\left[A^{\prime}, A^{\prime}\right]$ is well defined and we can write:

$$
\left[A^{\prime}, A^{\prime}\right]=\left[M+A-M^{\prime}, M+A-M^{\prime}\right]=\left[M-M^{\prime}, M-M^{\prime}\right]+[A, A]-2\left[A, M-M^{\prime}\right] .
$$


But, $M-M^{\prime}$ is a continuous local martingale and as $A$ is taken from the natural decomposition of $X$, we get: $\left[A, M-M^{\prime}\right]=0$, hence the desired result.

\section{Stability of weak Dirichlet processes under $C^{1}$ transformations}

Assume $X$ is a process of finite energy. Let us denote by $\mu$ the jump measure of $X$. Then $\sum_{s \leq} \Delta X_{s}^{2}$ can be written $\int_{0}^{\cdot} \int_{\mathbb{R}-\{0\}} x^{2} \mu(d s, d x)$. Since $E \sum_{s \leq T} \Delta X_{s}^{2}<\infty, X$ admits a Lévy system $\nu$ which is the predictable compensator of $\mu$; then the predictable increasing process $\int_{0}^{\cdot} \int_{\mathbb{R}-\{0\}} x^{2} \nu(d s, d x)$ is well defined and

$$
E \sum_{s \leq T} \Delta X_{s}^{2}=E\left[\int_{0}^{T} \int_{\mathbb{R}-\{0\}} x^{2} \mu(d s, d x)\right]=E\left[\int_{0}^{T} \int_{\mathbb{R}-\{0\}} x^{2} \nu(d s, d x)\right]<\infty .
$$

We begin with $C^{2}$ stability:

Theorem 3.1 Let $X=M+A$ be a weak Dirichlet process of finite energy and $F$ a $C^{2}$-real valued function with bounded derivatives $f$ and $f^{\prime}$. Then the process $\left(F\left(X_{t}\right)_{t \geq 0}\right)$ is a weak Dirichlet process of finite energy and the decomposition $F(X)=Y+\Gamma$ holds with the martingale part

$$
\begin{aligned}
Y_{t}= & F(0)+\int_{0}^{t} f\left(X_{s-}\right) d M_{s} \\
& +\int_{0}^{t} \int_{\mathbb{R}}\left(F\left(X_{s-}+x\right)-F\left(X_{s-}\right)-x f\left(X_{s-}\right)\right)(\mu-\nu)(d s, d x)
\end{aligned}
$$

and the predictable part

$$
\begin{aligned}
\Gamma_{t}= & \int_{0}^{t} f\left(X_{s-}\right) d A_{s}-1 / 2 \sum_{s \leq t} f^{\prime}\left(X_{s-}\right)\left(\Delta A_{s}\right)^{2}+1 / 2 \int_{0}^{t} f^{\prime}\left(X_{s}\right) d[M, M]_{s}^{c} \\
& +\int_{0}^{t} \int_{\mathbb{R}}\left(F\left(X_{s-}+x\right)-F\left(X_{s-}\right)-x f\left(X_{s-}\right)\right) \nu(d s, d x),
\end{aligned}
$$


where $(S) \int_{0} f\left(X_{s-}\right) d A_{s}$ is well defined as a limit in probability of Riemann sums. More precisely for every $t$

$\sum_{t_{i}^{n} \in D_{n}, t_{i}^{n} \leq t}\left(f\left(X_{t_{i}^{n}}\right)\left(A_{t_{i+1}^{n}}-A_{t_{i}^{n}}\right)+1 / 2 f^{\prime}\left(X_{t_{i}^{n}}\right)\left(A_{t_{i+1}^{n}}-A_{t_{i}^{n}}\right)^{2}\right) \stackrel{\mathbf{P}}{\longrightarrow}(S) \int_{0}^{t} f\left(X_{s-}\right) d A_{s}$.

Proof: Fix $t>0$. We use arguments from the paper [7] by Föllmer. For $\epsilon>0$ we define $J(1)=\left\{s \leq t ;\left|\Delta X_{s}\right|>\epsilon\right\}$. In the following, the elements of $D_{n}$ are, for short, written $t_{i}$ instead of $t_{i}^{n}$. Then

$\sum_{t_{i} \in D_{n}, t_{i} \leq t}\left(F\left(X_{t_{i+1}}\right)-F\left(X_{t_{i}}\right)\right)=\sum_{1}\left(F\left(X_{t_{i+1}}\right)-F\left(X_{t_{i}}\right)\right)+\sum_{2}\left(F\left(X_{t_{i+1}}\right)-\right.$ $\left.F\left(X_{t_{i}}\right)\right)$,

where $\sum_{1}$ denotes the sum (depending on $\omega \in \Omega$ ) of $t_{i} \in D_{n}, t_{i} \leq t$ such that $\left(t_{i}, t_{i+1}\right] \cap J(1) \neq \emptyset$ and $\sum_{2}$ the sum on the other $t_{i}$ 's.

Then by Taylor's formula

$$
\begin{aligned}
& \sum_{2}\left(F\left(X_{t_{i+1}}\right)-F\left(X_{t_{i}}\right)\right)=\sum_{2} f\left(X_{t_{i}}\right)\left(X_{t_{i+1}}-X_{t_{i}}\right) \\
& +1 / 2 \sum_{2} f^{\prime}\left(X_{t_{i}}\right)\left(X_{t_{i+1}}-X_{t_{i}}\right)^{2}+\sum_{2} r_{2}\left(X_{t_{i}}, X_{t_{i+1}}\right)
\end{aligned}
$$

where $r_{2}\left(X_{t_{i}}, X_{t_{i+1}}\right)=C_{i}^{\epsilon}\left(X_{t_{i+1}}-X_{t_{i}}\right)^{2}$ with $\max _{2}\left|C_{i}^{\epsilon}\right| \leq\left\|f^{\prime}\right\|$ and

$$
\lim _{\epsilon \downarrow 0} \limsup _{n \rightarrow \infty} P\left(\max _{2}\left|C_{i}^{\epsilon}\right|>\delta\right)=0, \quad \delta>0 .
$$

Hence

$$
\begin{aligned}
& \sum_{t_{i} \in D_{n}, t_{i} \leq t}\left(F\left(X_{t_{i+1}}\right)-F\left(X_{t_{i}}\right)\right)=\sum_{t_{i} \in D_{n}, t_{i} \leq t} f\left(X_{t_{i}}\right)\left(X_{t_{i+1}}-X_{t_{i}}\right) \\
& +1 / 2 \sum_{t_{i} \in D_{n}, t_{i} \leq t} f^{\prime}\left(X_{t_{i}}\right)\left(X_{t_{i+1}}-X_{t_{i}}\right)^{2}+\sum_{2} r_{2}\left(X_{t_{i}}, X_{t_{i+1}}\right) \\
& -\sum_{1}\left\{F\left(X_{t_{i+1}}\right)-F\left(X_{t_{i}}\right)-f\left(X_{t_{i}}\right)\left(X_{t_{i+1}}-X_{t_{i}}\right)-1 / 2 f^{\prime}\left(X_{t_{i}}\right)\left(X_{t_{i+1}}-X_{t_{i}}\right)^{2}\right\} \\
& =\sum_{t_{i} \in D_{n}, t_{i} \leq t} f\left(X_{t_{i}}\right)\left(M_{t_{i+1}}-M_{t_{i}}\right)+\sum_{t_{i} \in D_{n}, t_{i} \leq t} f\left(X_{t_{i}}\right)\left(A_{t_{i+1}}-A_{t_{i}}\right) \\
& +1 / 2 \sum_{t_{i} \in D_{n}, t_{i} \leq t} f^{\prime}\left(X_{t_{i}}\right)\left(A_{t_{i+1}}-A_{t_{i}}\right)^{2}+1 / 2 \sum_{t_{i} \in D_{n}, t_{i} \leq t} f^{\prime}\left(X_{t_{i}}\right)\left(M_{t_{i+1}}-M_{t_{i}}\right)^{2} \\
& +\sum_{t_{i} \in D_{n}, t_{i} \leq t} f^{\prime}\left(X_{t_{i}}\right)\left(M_{t_{i+1}}-M_{t_{i}}\right)\left(A_{t_{i+1}}-A_{t_{i}}\right)+\sum_{2} r_{2}\left(X_{t_{i}}, X_{t_{i+1}}\right)
\end{aligned}
$$




$$
\begin{aligned}
& -\sum_{1}\left\{F\left(X_{t_{i+1}}\right)-F\left(X_{t_{i}}\right)-f\left(X_{t_{i}}\right)\left(X_{t_{i+1}}-X_{t_{i}}\right)-1 / 2 f^{\prime}\left(X_{t_{i}}\right)\left(X_{t_{i+1}}-X_{t_{i}}\right)^{2}\right\} \\
& I_{1}^{n}+I_{2}^{n}+I_{3}^{n}+I_{4}^{n}+I_{5}^{n}+I_{6}^{n, \epsilon}+I_{7}^{n, \epsilon} .
\end{aligned}
$$

Now, note that by the definition of a stochastic integral we have $I_{1}^{n} \stackrel{\mathbf{P}}{\longrightarrow} \int_{0}^{t} f\left(X_{s-}\right) d M_{s}$.

The following simple lemma will be very useful in order to estimate the other terms.

Lemma 3.1 Assume that càdlàg processes $X$ and $Y$ admit a quadratic covariation $[X, Y]$ and that the sequence $\left\{\operatorname{Var}\left(S^{n}(X, Y)\right)_{T}\right\}$ is bounded in probability.

To càdlàg processes $Z$ and $U$ we associate the sequences $\left\{Z^{n}\right\}$ and $\left\{U^{n}\right\}$ of processes, where $Z^{n}$ and $U^{n}$ are the respective discretizations of $Z$ and $U$ along $D_{n}$; precisely $Z_{t}^{n}=Z_{t_{i}^{n}}, U_{t}^{n}=U_{t_{i}^{n}}$ when $t \in\left[t_{i}^{n}, t_{i+1}^{n}\right.$ [. Then, for every continuous real function $f, g$ and every $t$, holds the convergence:

$$
\int_{0}^{t} f\left(Z_{s-}^{n}\right) g\left(\Delta U_{s}^{n}\right) d S^{n}(X, Y)_{s} \stackrel{\mathbf{P}}{\longrightarrow} \int_{0}^{t} f\left(Z_{s-}\right) g\left(\Delta U_{s}\right) d[X, Y]_{s},
$$

where these integrals are Stieltjes integrals with respect to the processes $S^{n}(X, Y)$ or $[X, Y]$.

Proof of Lemma 3.1 From the proof of [2, Lemma 1.3] one can deduce that

$\int_{0} f\left(Z_{s-}^{n}\right) g\left(\Delta U_{s}^{n}\right) d S^{n}(X, Y)_{s} \stackrel{\mathrm{P}}{\longrightarrow} \int_{0} f\left(Z_{s-}\right) g\left(\Delta U_{s}^{n}\right) d[X, Y]_{s}$

in the (so-called $J_{1}$ ) Skorokhod topology (see e.g. [11]). Since for every

$f\left(Z_{t-}^{n}\right) g\left(\Delta U_{t}^{n}\right) \Delta S^{n}(X, Y)_{t} \stackrel{\mathbf{P}}{\longrightarrow} f\left(Z_{t-}\right) g\left(\Delta U_{t}\right) \Delta[X, Y]_{t}$,

the desired result follows from properties of the Skorokhod topology $J_{1}$.

It is clear by Lemma 3.1 that we have the convergences;

$$
\begin{aligned}
& I_{4}^{n} \stackrel{\mathrm{P}}{\longrightarrow} 1 / 2 \int_{0}^{t} f^{\prime}\left(X_{s-}\right) d[M, M]_{s}, \\
& I_{5}^{n} \stackrel{\mathrm{P}}{\longrightarrow} \int_{0}^{t} f^{\prime}\left(X_{s-}\right) d\left[M^{d}, A\right]_{s},
\end{aligned}
$$

where $M^{d}$ denotes purely discontinuous part of $M$.

Since $X$ is a process with finite energy, by (11) 
$\lim _{\epsilon \downarrow 0} \lim \sup _{n \rightarrow \infty} P\left(\left|I_{6}^{n, \epsilon}\right|>\delta\right)=0, \quad \delta>0$.

We observe also that $P$-almost surely there exists the limit

$$
\begin{aligned}
& \lim _{\epsilon \downarrow 0} \lim _{n \rightarrow \infty} I_{7}^{n, \epsilon}=\sum_{s \leq t}\left\{F\left(X_{s}\right)-F\left(X_{s-}\right)-f\left(X_{s-}\right) \Delta X_{s}-1 / 2 f^{\prime}\left(X_{s-}\right) \Delta X_{s}^{2}\right\} \\
& \quad=\sum_{s \leq t}\left\{F\left(X_{s}\right)-F\left(X_{s-}\right)-f\left(X_{s-}\right) \Delta X_{s}\right\} \\
& -1 / 2 \sum_{s \leq t}\left\{f^{\prime}\left(X_{s-}\right) \Delta M_{s}^{2}+\sum_{s \leq t} f^{\prime}\left(X_{s-}\right) \Delta A_{s}^{2}+2 \sum_{s \leq t} f^{\prime}\left(X_{s-}\right) \Delta M_{s}^{d} \Delta A_{s}\right\} .
\end{aligned}
$$

On the other hand it is obvious that $P$-almost surely

$$
\sum_{t_{i} \in D_{n}, t_{i} \leq t}\left(F\left(X_{t_{i+1}}\right)-F\left(X_{t_{i}}\right)\right) \rightarrow F\left(X_{t}\right)-F(0)
$$

and putting together all convergences, we deduce that $\left\{I_{2}^{n}+I_{3}^{n}\right\}$ is converging in probability and the limit we denote as $(S) \int_{0}^{t} f\left(X_{s-}\right) d A_{s}$. Observe also that

$$
\begin{aligned}
& \int_{0}^{t} f^{\prime}\left(X_{s-}\right) d[M, M]_{s}^{c}=\int_{0}^{t} f^{\prime}\left(X_{s-}\right) d[M, M]_{s}-\sum_{s \leq t} f^{\prime}\left(X_{s-}\right) \Delta M_{s}^{2} \\
& \text { and } \\
& \qquad \int_{0}^{t} f^{\prime}\left(X_{s-}\right) d\left[M^{d}, A\right]_{s}=\sum_{s \leq t} f\left(X_{s-}\right) \Delta M_{s} \Delta A_{s} .
\end{aligned}
$$

As a consequence we obtain the formula

$$
\begin{aligned}
F\left(X_{t}\right)= & F(0)+\int_{0}^{t} f\left(X_{s-}\right) d M_{s}+(S) \int_{0}^{t} f\left(X_{s-}\right) d A_{s} \\
& +1 / 2 \int_{0}^{t} f^{\prime}\left(X_{s-}\right) d[M, M]_{s}^{c}-1 / 2 \sum_{s \leq t} f^{\prime}\left(X_{s-}\right) \Delta A_{s}^{2} \\
& +\sum_{s \leq t}\left\{F\left(X_{s}\right)-F\left(X_{s-}\right)-\Delta X_{s} f\left(X_{s-}\right)\right\} .
\end{aligned}
$$

Now, writing

$$
\begin{aligned}
\sum_{s \leq t}\{ & \left.F\left(X_{s}\right)-F\left(X_{s-}\right)-\Delta X_{s} f\left(X_{s-}\right)\right\} \\
& =\int_{0}^{t} \int_{\mathbb{R}-\{0\}}\left(F\left(X_{s-}+x\right)-F\left(X_{s-}\right)-x f\left(X_{s-}\right)\right) \mu(d s, d x)
\end{aligned}
$$

and using the basic inequalities

$$
|F(y+x)-F(y)-x f(y)| \leq\left\|f^{\prime}\right\| x^{2}
$$

and

$$
|F(y+x)-F(y)-x f(y)| \leq 2\|f\||| x \mid,
$$


we get the decomposition

$$
\begin{aligned}
\sum_{s \leq .}\left\{F\left(X_{s}\right)-F\left(X_{s-}\right)-\Delta X_{s} f\left(X_{s-}\right)\right\} \\
=\int_{0} \int_{\mathbb{R}-\{0\}}\left(F\left(X_{s-}+x\right)-F\left(X_{s-}\right)-x f\left(X_{s-}\right)\right)(\mu-\nu)(d s, d x) \\
\quad+\int_{0}^{\cdot} \int_{\mathbb{R}-\{0\}}\left(F\left(X_{s-}+x\right)-F\left(X_{s-}\right)-x f\left(X_{s-}\right)\right) \nu(d s, d x)
\end{aligned}
$$

where

$$
\int_{0} \int_{\mathbb{R}-\{0\}}\left(F\left(X_{s-}+x\right)-F\left(X_{s-}\right)-x f\left(X_{s-}\right)(\mu-\nu)(d s, d x)\right.
$$

is a square integrable purely discontinuous martingale, which we will denote by $L$ and

$$
\int_{0} \int_{\mathbb{R}-\{0\}}\left(F\left(X_{s-}+x\right)-F\left(X_{s-}\right)-x f\left(X_{s-}\right)\right) \nu(d s, d x)
$$

is an increasing predictable square integrable process.

Then we get the decomposition $F(X)=.F(0)+Y$. $+\Gamma$, as written in the statement of Theorem 3.1.

It remains to prove that, for every continuous local martingale $N$, holds the equality: $[\Gamma, N]=0$.

First note that

$$
\begin{aligned}
& \sum_{t_{i} \in D_{n}, t_{i} \leq t}\left(\Gamma_{t_{i+1}}-\Gamma_{t_{i}}\right)\left(N_{t_{i+1}}-N_{t_{i}}\right) \\
&=\sum_{t_{i} \in D_{n}, t_{i} \leq t} \int_{t_{i}}^{t_{i+1}}\left(f\left(X_{s-}\right)-f\left(X_{t_{i}}\right)\right) d M_{s}\left(N_{t_{i+1}}-N_{t_{i}}\right) \\
& \quad-\sum_{t_{i} \in D_{n}, t_{i} \leq t}\left(L_{t_{i+1}}-L_{t_{i}}\right)\left(N_{t_{i+1}}-N_{t_{i}}\right) \\
& \quad+\sum_{t_{i} \in D_{n}, t_{i} \leq t} f\left(X_{t_{i}}\right)\left(A_{t_{i+1}}-A_{t_{i}}\right)\left(N_{t_{i+1}}-N_{t_{i}}\right) \\
& \quad+1 / 2 \sum_{t_{i} \in D_{n}, t_{i} \leq t} f^{\prime}\left(X_{t_{i}}\right)\left(A_{t_{i+1}}-A_{t_{i}}\right)^{2}\left(N_{t_{i+1}}-N_{t_{i}}\right) \\
& \quad+1 / 2 \sum_{t_{i} \in D_{n}, t_{i} \leq t} f^{\prime}\left(X_{t_{i}}\right)\left(M_{t_{i+1}}-M_{t_{i}}\right)^{2}\left(N_{t_{i+1}}-N_{t_{i}}\right) \\
&+\sum_{t_{i} \in D_{n}, t_{i} \leq t} f^{\prime}\left(X_{t_{i}}\right)\left(M_{t_{i+1}}-M_{t_{i}}\right)\left(A_{t_{i+1}}-A_{t_{i}}\right)\left(N_{t_{i+1}}-N_{t_{i}}\right)
\end{aligned}
$$




$$
\begin{gathered}
+\sum_{2} r_{2}\left(X_{t_{i}}, X_{t_{i+1}}\right)\left(N_{t_{i+1}}-N_{t_{i}}\right) \\
-\sum_{1}\left\{F\left(X_{t_{i+1}}\right)-F\left(X_{t_{i}}\right)-f\left(X_{t_{i}}\right)\left(X_{t_{i+1}}-X_{t_{i}}\right)\right. \\
\left.\quad-1 / 2 f^{\prime}\left(X_{t_{i}}\right)\left(X_{t_{i+1}}-X_{t_{i}}\right)^{2}\right\}\left(N_{t_{i+1}}-N_{t_{i}}\right) \\
=I_{1}^{n}+I_{2}^{n}+I_{3}^{n}+I_{4}^{n}+I_{5}^{n}+I_{6}^{n}+I_{7}^{n, \epsilon}+I_{8}^{n, \epsilon}
\end{gathered}
$$

Clearly,

$$
\left|I_{1}^{n}\right| \leq\left(\sum_{t_{i} \in D_{n}, t_{i} \leq t}\left(\int_{t_{i}}^{t_{i+1}}\left(f\left(X_{s-}\right)-f\left(X_{t_{i}}\right)\right) d M_{s}\right)^{2}\right)^{1 / 2}\left(\sum_{t_{i} \in D_{n}, t_{i} \leq t}\left(N_{t_{i+1}}-N_{t_{i}}\right)^{2}\right)^{1 / 2},
$$

where by the definition of the stochastic integral

$$
\begin{aligned}
E \sum_{t_{i} \in D_{n}, t_{i} \leq t} & \left(\int_{t_{i}}^{t_{i+1}}\left(f\left(X_{s-}\right)-f\left(X_{t_{i}}\right)\right) d M_{s}\right)^{2} \\
= & E \sum_{t_{i} \in D_{n}, t_{i} \leq t} \int_{t_{i}}^{t_{i+1}}\left(f\left(X_{s-}\right)-f\left(X_{t_{i}}\right)\right)^{2} d[M, M]_{s} \rightarrow 0 .
\end{aligned}
$$

On the other hand by Lemma 3.1

$$
\begin{aligned}
& I_{2}^{n} \stackrel{\mathbf{P}}{\longrightarrow}[L, N]_{t}=0, \\
& I_{3}^{n} \stackrel{\mathbf{P}}{\longrightarrow} \int_{0}^{t} f\left(X_{s-}\right) d[A, N]_{s}=0 \\
& I_{4}^{n} \stackrel{\mathbf{P}}{\longrightarrow} 1 / 2 \int_{0}^{t} f^{\prime}\left(X_{s-}\right) \Delta A_{s} d[A, N]_{s}=0 \\
& I_{5}^{n} \stackrel{\mathbf{P}}{\longrightarrow} 1 / 2 \int_{0}^{t} f^{\prime}\left(X_{s-}\right) \Delta N_{s} d[M, M]_{s}=0
\end{aligned}
$$

and

$$
I_{6}^{n} \stackrel{\mathbf{P}}{\longrightarrow} \int_{0}^{t} f^{\prime}\left(X_{s-}\right) \Delta M_{s} d[A, N]_{s}=0 .
$$

Finally, for every $\epsilon>0$

$$
I_{6}^{n, \epsilon} \stackrel{\mathrm{P}}{\longrightarrow} 0 \quad \text { and } \quad I_{7}^{n, \epsilon} \rightarrow 0, P-\text { a.s. }
$$

and the proof of Theorem 3.1 is completed. 
Corollary 3.1 Let $X=M+A$ be a weak Dirichlet process of finite energy admitting a quadratic variation and $F$ be a $C^{2}$-real valued function with bounded derivatives $f$ and $f^{\prime}$. Then the process $\left(F\left(X_{t}\right)_{t \geq 0}\right)$ is a weak Dirichlet process of finite energy admitting a qudratic variation and the decomposition $F(X)=Y+\Gamma$ holds with the martingale part

$$
\begin{aligned}
Y_{t}= & F(0)+\int_{0}^{t} f\left(X_{s-}\right) d M_{s} \\
& +\int_{0}^{t} \int_{\mathbb{R}}\left(F\left(X_{s-}+x\right)-F\left(X_{s-}\right)-x f\left(X_{s-}\right)\right)(\mu-\nu)(d s, d x)
\end{aligned}
$$

and the predictable part

$$
\begin{aligned}
\Gamma_{t}= & \int_{0}^{t} f\left(X_{s-}\right) d A_{s}+1 / 2 \int_{0}^{t} f^{\prime}\left(X_{s}\right) d[X, X]_{s}^{c} \\
& \quad+\int_{0}^{t} \int_{\mathbb{R}}\left(F\left(X_{s-}+x\right)-F\left(X_{s-}\right)-x f\left(X_{s-}\right)\right) \nu(d s, d x) .
\end{aligned}
$$

Proof: By Theorem 2.3(i), $A$ admits integrable quadratic variation $[A, A]$ and due to Lemma 3.1

$$
1 / 2 \sum_{t_{i} \in D_{n}, t_{i} \leq t} f\left(X_{t_{i}}\right)\left(A_{t_{i+1}}-A_{t_{i}}\right)^{2} \stackrel{\mathbf{P}}{\longrightarrow} 1 / 2 \int_{0}^{t} f\left(X_{s-}\right) d[A, A]_{s} .
$$

Since $[X, X]^{c}=[M, M]^{c}+[A, A]^{c}$, it is clear that

$$
\begin{aligned}
1 / 2 \int_{0}^{t} f\left(X_{s-}\right) d[A, A]_{s}-1 / 2 \sum_{s \leq t} f\left(X_{s-}\right) \Delta A_{s}^{2}+1 / 2 \int_{0}^{t} f\left(X_{s-}\right) d[M, M]_{s} \\
=1 / 2 \int_{0}^{t} f\left(X_{s_{-}}\right) d\left([A, A]_{s}^{c}+[M, M]^{c}\right)=1 / 2 \int_{0}^{t} f\left(X_{s-}\right) d[X, X]_{s}^{c}
\end{aligned}
$$

and the decomposition $F\left(X_{t}\right)=F(0)+Y_{t}+\Gamma_{t}$ in the statement of Corollary 3.1 is a consequence of Theorem 3.1.

Finally, by the Theorem from [7, page 144] we obtain that also $F(X)$ admits a quadratic variation, which completes the proof.

Theorem 3.2 Let $X=M+A$ be a weak Dirichlet process of finite energy and $F$ a $C^{1}$-real valued function with bounded derivative $f$.

Then the process $\left(F\left(X_{t}\right)_{t \geq 0}\right)$ is a weak Dirichlet process of finite energy and the decomposition $F(X)=Y+\Gamma$ holds with the martingale part

$$
\begin{aligned}
Y_{t}= & F(0)+\int_{0}^{t} f\left(X_{s-}\right) d M_{s} \\
& +\int_{0}^{t} \int_{\mathbb{R}}\left(F\left(X_{s-}+x\right)-F\left(X_{s-}\right)-x f\left(X_{s-}\right)\right)(\mu-\nu)(d s, d x)
\end{aligned}
$$


Remark 3.1 This theorem has formally almost the same statement as Theorem 3.1. However, we have not here any explicit formula for $\Gamma$. The delicate point here is the behaviour of the sum

$$
\sum_{s \leq t}\left(F\left(X_{s}\right)-F\left(X_{s-}\right)-\Delta X_{s} f\left(X_{s-}\right)\right)
$$

which is not necessarily absolutely convergent and which does not define a process with finite variation.

Proof of Theorem 3.2: We consider a sequence $\left(F^{p}\right)_{p \in \mathbb{N}}$ of $C^{2}$ real functions such that $\left\|F-F^{p}\right\|+\left\|f-f^{p}\right\| \rightarrow 0$, when $p \rightarrow \infty$. Using Theorem 3.1 we can write

$$
F^{p}\left(X_{t}\right)=F^{p}(0)+Y_{t}^{p}+\Gamma_{t}^{p}
$$

where

$$
Y_{t}^{p}=\int_{0}^{t} f^{p}\left(X_{s-}\right) d M_{s}+L_{t}^{p}
$$

with

$$
L_{t}^{p}=\int_{0}^{t} \int_{\mathbb{R}-\{0\}}\left(F^{p}\left(X_{s-}+x\right)-F^{p}\left(X_{s-}\right)-x f^{p}\left(X_{s-}\right)\right)(\mu-\nu)(d s, d x) .
$$

The sequence $\left\{\int_{0} f^{p}\left(X_{s-}\right) d M_{s}+L^{p}\right\}_{p \in \mathbb{N}}$ is a Cauchy sequence in the space $H^{2}$ of square integrable martingales, hence the limiting martingale exists and has the form $\int_{0} f\left(X_{s-}\right) d M_{s}+L$ :

Actually, for $p, q$ integers

$$
\begin{gathered}
\left\|Y^{p}-Y^{q}\right\|_{H^{2}} \leq E\left(\int_{0}^{T}\left(f^{p}\left(X_{s-}\right)-f^{q}\left(X_{s-}\right)\right)^{2} d[M, M]_{s}\right) \\
+E\left[\int _ { 0 } ^ { T } \int _ { \mathbb { R } - \{ 0 \} } \left(F^{p}\left(X_{s-}+x\right)-F^{p}\left(X_{s-}\right)-x f^{p}\left(X_{s-}\right)\right.\right. \\
\left.\left.-F^{q}\left(X_{s-}+x\right)+F^{q}\left(X_{s-}\right)+x f^{q}\left(X_{s-}\right)\right)^{2} \nu(d s, d x)\right] \\
\leq\left\|F^{p}-F^{q}\right\|^{2} E[[M, M]]+2\left\|f^{p}-f^{q}\right\|^{2} E[[M, M]] .
\end{gathered}
$$

Now we write:

$$
\Gamma_{t}^{p}=F^{p}\left(X_{t}\right)-F^{p}(0)-\int_{0}^{t} f^{p}\left(X_{s-}\right) d M_{s}-L_{t}^{p} .
$$

Clearly the sequence of predictable processes $\left(\Gamma^{p}\right)$ converges uniformly in probability and its limit (i.e. the process $\Gamma$ ) has to be also predictable. 
It remains to prove that $[\Gamma, N]=0$ for every continuous local martingale $N$.

Fix $t$. In the sequel use the notations from the proof of Theorem 3.1.

By Taylor's formula

$$
\sum_{2}\left(F\left(X_{t_{i+1}}\right)-F\left(X_{t_{i}}\right)\right)=\sum_{2} f\left(X_{t_{i}}\right)\left(X_{t_{i+1}}-X_{t_{i}}\right)+\sum_{2} r_{1}\left(X_{t_{i}}, X_{t_{i+1}}\right),
$$

where $r_{1}\left(X_{t_{i}}, X_{t_{i+1}}\right)=C_{i}^{\epsilon}\left(X_{t_{i+1}}-X_{t_{i}}\right)$ satisfy $\left|C_{i}^{\epsilon}\right| \leq\|f\|$ and

$$
\lim _{\epsilon \downarrow 0} \limsup _{n \rightarrow \infty} P\left(\max _{2}\left|C_{i}^{\epsilon}\right|>\delta\right)=0, \quad \delta>0 .
$$

Therefore,

$$
\begin{aligned}
& \sum_{t_{i} \in D_{n}, t_{i} \leq t}\left(\Gamma_{t_{i+1}}-\Gamma_{t_{i}}\right)\left(N_{t_{i+1}}-N_{t_{i}}\right) \\
& =\sum_{t_{i} \in D_{n}, t_{i} \leq t} \int_{t_{i}}^{t_{i+1}} f\left(X_{t_{i}}\right)-f\left(X_{s-}\right) d M_{s}\left(N_{t_{i+1}}-N_{t_{i}}\right) \\
& \quad-\sum_{t_{i} \in D_{n}, t_{i} \leq t}\left(L_{t_{i+1}}-L_{t_{i}}\right)\left(N_{t_{i+1}}-N_{t_{i}}\right) \\
& \quad+\sum_{t_{i} \in D_{n}, t_{i} \leq t} f\left(X_{t_{i}}\right)\left(A_{t_{i+1}}-A_{t_{i}}\right)\left(N_{t_{i+1}}-N_{t_{i}}\right) \\
& \quad+\sum_{2} r_{1}\left(X_{t_{i}}, X_{t_{i+1}}\right)\left(N_{t_{i+1}}-N_{t_{i}}\right) \\
& \quad-\sum_{1}\left\{F\left(X_{t_{i+1}}\right)-F\left(X_{t_{i}}\right)-f\left(X_{t_{i}}\right)\left(X_{t_{i+1}}-X_{t_{i}}\right)\left(N_{t_{i+1}}-N_{t_{i}}\right)\right\} \\
& =I_{1}^{n}+I_{2}^{n}+I_{3}^{n}+I_{4}^{n, \epsilon}+I_{5}^{n, \epsilon} .
\end{aligned}
$$

Clearly, first three sums tend to 0 analogously to the proof of Theorem 3.1. Next,

$$
\lim _{\epsilon \downarrow 0} \limsup _{n \rightarrow \infty} P\left(\left|I_{4}^{n, \epsilon}\right|>\delta\right)=0, \text { quad } \delta>0
$$

and for every $\epsilon>0$

$$
I_{5}^{n, \epsilon} \rightarrow 0, P-a . s .
$$

which, completes the proof of Theorem 3.2. 
Corollary 3.2 Let $X=M+A$ be a weak Dirichlet process of finite energy admitting a quadratic variation process and $F$ a $C^{1}$-real valued function with bounded derivative $f$. Then the process $\left(F\left(X_{t}\right)_{t \geq 0}\right)$ is a weak Dirichlet process of finite energy admitting a quadratic variation and the decomposition $F(X)=Y+\Gamma$ holds with the martingale part

$$
\begin{aligned}
Y_{t}= & F(0)+\int_{0}^{t} f\left(X_{s-}\right) d M_{s} \\
& +\int_{0}^{t} \int_{\mathbb{R}}\left(F\left(X_{s-}+x\right)-F\left(X_{s-}\right)-x f\left(X_{s-}\right)\right)(\mu-\nu)(d s, d x)
\end{aligned}
$$

The quadratic variation process of $F\left(X_{t}\right)_{t}$ is given by

$$
[F(X), F(X)]_{t}=\int_{0}^{t}\left(f\left(X_{s}\right)\right)^{2} d[M, M]_{s}^{c}+\int_{0}^{t}\left(f\left(X_{s}\right)\right)^{2} d[A, A]_{s}^{c}+\sum_{0 \leq s \leq t}\left(F\left(X_{s}\right)-F\left(X_{s-}\right)\right)^{2} .
$$

Proof: Follows easily from Theorem 3.2, [7, Theorem, page 144] and the equality $[X, X]^{c}=[M, M]^{c}+[A, A]^{c}$.

We are able to prove a version of Theorem 3.2 for weak Dirichlet processes also with infinite energy. However, in this case we have restricted our attention to processes with a continuous predictable part.

Theorem 3.3 Let $X=M+A$ be a weak Dirichlet process with continuous predictable part $A$ and $F$ a $C^{1}$ real-valued function with bounded derivative $f$.

Then the process $\left(F\left(X_{t}\right)_{t \geq 0}\right)$ is a weak Dirichlet process and the decomposition $F(X)=Y+\Gamma$ holds with the martingale part

$$
\begin{aligned}
Y_{t}= & F(0)+\int_{0}^{t} f\left(X_{s-}\right) d M_{s} \\
& +\int_{0}^{t} \int_{\mathbb{R}}\left(F\left(X_{s-}+x\right)-F\left(X_{s-}\right)-x f\left(X_{s-}\right)\right)(\mu-\nu)(d s, d x)
\end{aligned}
$$

Proof: We consider a sequence $\left(F^{p}\right)_{p \in \mathbb{N}}$ of $C^{2}$ real functions such that locally on compact sets $\left\|F-F^{p}\right\|+\left\|f-f^{p}\right\| \rightarrow 0$, when $p \rightarrow \infty$. Let $A^{p}$ be a sequence of continuous processes with finite variation such that

$$
\sup _{t \leq T}\left|A_{t}^{p}-A_{t}\right| \stackrel{\mathbf{P}}{\longrightarrow} 0 .
$$


Using classical Itô's formula for the semimartingale $X^{p}=M+A^{p}$ we can write

$$
F^{p}\left(X_{t}^{p}\right)=F^{p}(0)+Y_{t}^{p}+\Gamma_{t}^{p}
$$

where

$$
Y_{t}^{p}=\int_{0}^{t} f^{p}\left(X_{s-}^{p}\right) d M_{s}+L_{t}^{p}
$$

with

$$
L_{t}^{p}=\int_{0}^{t} \int_{\mathbb{R}-\{0\}}\left(F^{p}\left(X_{s-}^{p}+x\right)-F^{p}\left(X_{s-}^{p}\right)-x f^{p}\left(X_{s-}^{p}\right)\right)(\mu-\nu)(d s, d x) .
$$

Similarly to the proof of Theorem 3.2 , we check that

$$
\sup _{t \leq T}\left|Y^{p}-Y_{t}\right| \stackrel{\mathbf{P}}{\longrightarrow} 0 .
$$

On the other hand it is clear that

$$
\sup _{t \leq T}\left|F^{p}\left(X_{t}^{p}\right)-F\left(X_{t}\right)\right| \stackrel{\mathbf{P}}{\longrightarrow} 0
$$

which implies that $\Gamma$ as a uniform limit of predictable processes is also predictable. Finally, by the same arguments as in the proof of Theorem 3.2 we prove that $[\Gamma, N]=0$ for every continuous local martingale $N$.

\section{Weak Dirichlet processes and generalized mar- tingale convolutions}

In this section we deal with processes $X$ such that

$$
X_{t}=\int_{0}^{t} G(t, s) d L_{s}
$$

where $L$ is a quasileft continuous square integrable martingale, and $G$ a real valued deterministic function of $(s, t)$.

Let us consider the following hypotheses on $G$.

$\left(H_{0}\right):(t, s) \rightarrow G(t, s)$ is continuous on $\{(s, t): 0<s \leq t \leq T\}$. 
$\left(H_{1}\right):$ For all $s, t \rightarrow G(t, s)$ has a bounded energy on $\left.] s, T\right]$ that is

$$
V_{2}^{2}(G)((s, T], s)=\sup _{n} \sum_{t_{i} \in D_{n}, t_{i} \geq s}\left(G\left(t_{i+1}, s\right)-G\left(t_{i}, s\right)\right)^{2}<\infty .
$$

$\left(H_{2}\right)$ :

$$
\left.\left.E\left[\int_{0}^{T} V_{2}^{2}(G)(] s, T\right]\right) d[L, L]_{s}\right]<\infty
$$

$\left(H_{3}\right): \quad \quad E\left[\int_{0}^{T} \Gamma^{2}(s) d[L, L]_{s}\right]<\infty$

where $\Gamma^{2}(s)=\sup _{t \leq T} G^{2}(t, s)$.

Remark 4.1 Errami and Russo ([5]) use, instead of $\left(H_{0}\right)$ a slightly more restrictive assumption, namely: $\left(H_{0^{+}}\right):(t, s) \rightarrow G(t, s)$ is continuous on $\{(s, t): 0 \leq s \leq t \leq T\}$.

Note that $\left(H_{0^{+}}\right)$, implies $\left(H_{3}\right)$. Actually $\Gamma^{2}$ is continuous and bounded.

If $t \rightarrow G(t, s)$ admits a quadratic variation on $(s, T]$ along $\left(D_{n}\right)$, then $\left(H_{1}\right)$ is satisfied.

We shall extend $G$ to the square $[0, T]^{2}$ by setting $G(t, s)=0$ if $s>t$.

Theorem 4.1 If $X$ meets (12) and if $G$ satisfies $\left(H_{0}\right),\left(H_{1}\right),\left(H_{2}\right),\left(H_{3}\right)$, then

(i) $X$ is a continuous in probability process with finite energy and has an optional modification,

(ii) Let us assume that $X$ has a.s. càdlàg trajectories, then $X$ is a weak Dirichlet process with natural decomposition $X=M+A$, such that if $M^{n}$ is defined as in (3), then for every $t \leq T$, $\left|M_{t}^{n}-M_{t}\right| \rightarrow 0$ in $\mathbf{L}^{2}$.

Proof: The proof will be given in several steps.

Lemma 4.1 $X$ is a continuous in probability process with finite energy. 
Proof of Lemma 4.1: First of all, from $\left(\mathrm{H}_{2}\right)$ and $\left(\mathrm{H}_{3}\right)$, for every $t \leq T$ $X_{t}$ is an $\mathcal{F}_{t}$-measurable square integrable random variable.

Let us write

$$
X_{t_{i+1}}-X_{t_{i}}=\int_{0}^{t_{i}}\left(G\left(T_{i+1}, s\right)-G\left(t_{i}, s\right)\right) d L_{s}+\int_{t_{i}}^{t_{i+1}} G\left(t_{i+1}, s\right) d L_{s} .
$$

Since $L$ is a square integrable martingale, we get:

$$
\begin{aligned}
& E\left(\sum_{t_{i} \in D_{n}}\left(X_{t_{i+1}}-X_{t_{i}}\right)^{2}\right) \\
& \leq 2 E\left(\sum_{t_{i} \in D_{n}}\left(\int_{0}^{t_{i}}\left(G\left(t_{i+1}, s\right)-G\left(t_{i}, s\right)\right) d L_{s}\right)^{2}\right)+2 E\left(\sum_{t_{i} \in D_{n}}\left(\int_{t_{i}}^{t_{i+1}} G\left(t_{i+1}, s\right) d L_{s}\right)^{2}\right) \\
& \leq 2 E\left(\sum_{t_{i} \in D_{n}} \int_{0}^{t_{i}}\left(G\left(t_{i+1}, s-G\left(t_{i}, s\right)\right)^{2} d[L, L]_{s}\right)+2 E\left(\sum_{t_{i} \in D_{n}} \int_{t_{i}}^{t_{i+1}}\left(G\left(t_{i+1}, s\right)\right)^{2} d[L, L]_{s}\right.\right. \\
& =2 E\left(I_{1}^{n}\right)+2 E\left(I_{2}^{n}\right) .
\end{aligned}
$$

By simple calculations

$$
\begin{aligned}
I_{1}^{n} & =\sum_{t_{i} \in D_{n}} \sum_{k=1}^{i} \int_{t_{k-1}}^{t_{k}}\left(G\left(t_{i+1}, s\right)-G\left(t_{i}, s\right)\right)^{2} d[L, L]_{s} \\
& =\sum_{t_{k} \in D_{n}} \int_{t_{k-1}}^{t_{k}} \sum_{i>k}\left(G\left(t_{i+1}, s\right)-G\left(t_{i}, s\right)\right)^{2} d[L, L]_{s} \\
& \leq \sum_{t_{k} \in D_{n}} \int_{t_{k-1}}^{t_{k}} V_{2}^{2}(G)((s, T], s) d[L, L]_{s},
\end{aligned}
$$

and

$$
\begin{aligned}
I_{2}^{n} & \leq \sum_{t_{i} \in D_{n}} \int_{t_{i}}^{t_{i+1}}\left(\Gamma^{2}(s) d[L, L]_{s}\right. \\
& \leq \int_{0}^{T} \Gamma^{2}(s) d[L, L]_{s} .
\end{aligned}
$$

Therefore

$$
\begin{aligned}
\sup _{n} E\left(\sum_{t_{i} \in D_{n}}\left(X_{t_{i+1}}-X_{t_{i}}\right)^{2}\right. & \leq 2 E \int_{0}^{T} V_{2}^{2}(G)((s, T], s) d[L, L]_{s}+2 E \int_{0}^{T}(\Gamma(s))^{2} d[L, L]_{s} \\
& <\infty .
\end{aligned}
$$

This proves that $X$ has a finite energy. 
Now, let us take $s, t$ such that $0 \leq s<t \leq T$. We get:

$$
E\left[\left(X_{t}-X_{s}\right)^{2}\right] \leq 2 E \int_{0}^{s}(G(t, u)-G(s, u))^{2} d[L, L]_{u}+2 E \int_{s}^{t}(G(t, u))^{2} d[L, L]_{u} .
$$

Since $L$ is continuous in probability, so is $[L, L]$. Under $\left(H_{3}\right)$,

$$
2 E \int_{s}^{t}(G(t, u))^{2} d[L, L]_{u} \rightarrow 0, \quad \text { as } t \rightarrow s,
$$

then by continuity of $t \rightarrow G(t, s)$ and dominated convergence,

$$
E \int_{0}^{s}(G(t, u)-G(s, u))^{2} d[L, L]_{u} \rightarrow 0 .
$$

The continuity in probability of the process $X$ follows.

At last, since the process $X$ is $\mathcal{F}_{t}$-adapted and continuous in probability, it admits an optional modification that we shall denote again by $X$ : see for example [12] pp 230-231, where Théorème 5 bis is given for a progressively measurable modification, but the sequence of approximating processes introduced in the proof is càdlàg hence optional (see also below the proof of the existence of a predictable modification of the process $A$ ).

Therefore Lemma 4.1 is proven.

Lemma 4.2 Let us consider the decomposition:

$X_{t}=A_{t}^{n}+M_{t}^{n}$,

where as in (3),

$$
M_{t}^{n}=\sum_{t_{i}^{n} \in D_{n}, t_{i}^{n} \leq t}\left[X_{t_{i}^{n}}-E\left[X_{t_{i}^{n}} / \mathcal{F}_{t_{i-1}^{n}}\right]\right] .
$$

Then $X$ admits a modification with a decomposition $X_{t}=A_{t}+M_{t}$, where $M$ is the square integrable martingale $M_{t}=\int_{0}^{t} G(s, s) d L_{s}$, and $A$ a predictable process, such that:

(i) for every $t \leq T$,

$$
\left|M_{t}^{n}-M_{t}\right| \rightarrow 0 \text { in } \mathbf{L}^{2},
$$

(ii) for every $t \leq T,\left|A_{t}^{n}-A_{t}\right| \rightarrow 0$ in $\mathbf{L}^{2}$, 
(iii) for every continuous local martingale $N,[A, N]=0$.

Proof of Lemma 4.2: For every $t_{i}, t_{i+1}$ we have

$$
E\left[X_{t_{i+1}}-X_{t_{i}} \mid \mathcal{F}_{t_{i}}\right]=\int_{0}^{t_{i}}\left(G\left(t_{i+1}, s\right)-G\left(t_{i}, s\right)\right) d L_{s}
$$

hence for $t \in[0, T]$

$$
M_{t}^{n}=\sum_{t_{i+1} \leq t} \int_{t_{i}}^{t_{i+1}} G\left(t_{i+1}, s\right) d L_{s}=\int_{0}^{\rho^{n}(t)} G^{n}(s) d L_{s},
$$

where $G^{n}(s)=G\left(t_{i+1}, s\right)$ for $s \in\left(t_{i}, t_{i+1}\right]$ and $\rho^{n}(t)=\max \left\{t_{i}: t_{i} \leq t\right\}$. Note that $\rho^{n}(t) \rightarrow t$ as $n \rightarrow \infty$.

Define $M_{t}=\int_{0}^{t} G(s, s) d L_{s}$. By $\left(H_{2}\right)$ and $\left(H_{3}\right)$, for every $\varepsilon>0$

$$
E\left[\left(M_{\varepsilon}\right)^{2}\right]=E \int_{0}^{\varepsilon} G^{2}(s, s) d[L, L]_{s} \leq E \int_{0}^{\varepsilon} \Gamma^{2}(s) d[L, L]_{s}
$$

and this last expression tends to 0 when $\varepsilon$ tends to 0 .

Similarly, when $\varepsilon \rightarrow 0$

$$
\sup _{n} E\left[\left(M_{\varepsilon}^{n}\right)^{2}=\sup _{n} E \int_{0}^{\varepsilon}\left(G^{n}(s)\right)^{2} d[L, L]_{s} \rightarrow 0 .\right.
$$

Now, let us fix $\varepsilon>0$ and belonging to $D_{n}$. Since $(t, s) \rightarrow G(t, s)$ on $\{(s, t): \varepsilon \leq s<t \leq T\}$ is continuous, then it is uniformly continuous and therefore:

$$
\sup _{\varepsilon \leq s \leq T}\left|G^{n}(s)-G(s, s)\right| \rightarrow 0 .
$$

Hence

$$
\begin{aligned}
E\left[\left(M_{t}^{n}-M_{\varepsilon}^{n}-M_{t}+M_{\varepsilon}\right)^{2}\right] \leq & 2 E\left(\int_{\varepsilon}^{\rho^{n}(t)}\left(G^{n}(s)-G(s, s)\right) d L_{s}\right)^{2} \\
& +2 E\left(\int_{\rho^{n}(t)}^{t} G(s, s) d L_{s}\right)^{2} \\
\leq & 8 E \int_{\varepsilon}^{1}\left(G^{n}(s)-G(s, s)\right)^{2} d[L, L]_{s} \\
& +2 E \int_{\rho^{n}(t)}^{t} G^{2}(s, s) d[L, L]_{s} .
\end{aligned}
$$


The first term tends to 0 when $n \rightarrow \infty$ because $G^{n}$ converges uniformly to $G$ on $[\varepsilon, T]$. Now, the continuity in probability of $L$ implies the continuity in probability of $M$ on $[0, T]$, hence the second term tends to 0 when $n \rightarrow \infty$.

Note that for every $n$

$$
A_{t}^{n}=\sum_{t_{i}^{n} \in D_{n}, t_{i}^{n} \leq t} \mathbf{E}\left[X_{t_{i}^{n}}-X_{t_{i-1}^{n}} / \mathcal{F}_{t_{i-1}^{n}}\right]
$$

and $A=X-M . A^{n}$ is a predictable process and we have $X_{\rho^{n}(t)}=A_{\rho^{n}(t)}^{n}+$ $M_{\rho^{n}(t)}^{n}$.

As $X$ is continuous in probability, for every $t \leq T, X_{\rho^{n}(t)} \rightarrow X_{t}$ in $\mathbf{L}^{2}$; moreover, $M_{t}^{n}=M_{\rho^{n}(t)}^{n}$ and $A_{t}^{n}=A_{\rho^{n}(t)}^{n}$ : we deduce that $A_{t}^{n} \rightarrow A_{t}$ in $\mathbf{L}^{2}$ for every $t$.

It follows that $A$ is also adapted and continuous in probability.

Our decomposition $X=M+A$ coincides with the Graversen-Rao decomposition of Theorem 2.1. But in Theorem 2.1, it is assumed that $X$ is càdlàg; here it is not the case, it is necessary to check that $A$ admits a predictable modification.

Actually, since $A$ is continuous in probability on the interval $[0,1]$, one can find a subsequence $\{n(k)\}_{k \geq 1}$ such that for every $t \in[0,1], \bar{A}^{n(k)} \rightarrow A_{t}$ a.s. when $k \rightarrow \infty$, whith $\bar{A}_{t}^{n}=\sum_{i} 1_{\left(t_{i}^{n}, t_{i+1}^{n}\right]}(t) A_{t_{i}^{n}}$ and $A_{0}^{n}=A_{0}$. Since every $\bar{A}^{n}$ is a step process adapted and left continuous, it is predictable, and the process $A^{\prime}$ defined by $A_{t}^{\prime}=\lim \sup _{k} \bar{A}_{t}^{n(k)}$ is also predictable. But for every $t, A_{t}^{\prime}=A_{t}$ a.s. So, we shall suppose now that $A=A^{\prime}$, and $X=A^{\prime}+M$.

Proof of (iii): Let $N$ be a continuous local martingale. Using localization arguments, we will assume that $N$ is a square integrable martingale. For every $t$ we can write:

$$
\begin{aligned}
\sum_{t_{i} \in D_{n}, t_{i} \leq t}\left(N_{t_{i+1}}-N_{t_{i}}\right)\left(A_{t_{i+1}}-A_{t_{i}}\right) \\
=\sum_{t_{i} \in D_{n}, t_{i} \leq t}\left(N_{t_{i+1}}-N_{t_{i}}\right) \int_{0}^{t_{i}}\left(G\left(t_{i+1}, s\right)-G\left(t_{i}, s\right)\right) d L_{s} \\
\quad+\sum_{t_{i} \in D_{n}, t_{i} \leq t}\left(N_{t_{i+1}}-N_{t_{i}}\right) \int_{t_{i}}^{t_{i+1}}\left(G\left(t_{i+1}, s\right)-G(s, s)\right) d L_{s} \\
=I_{1}^{n}+I_{2}^{n}
\end{aligned}
$$

Since $N$ is a martingale, using the B-D-G inequality and Schwarz's inequality 
we get:

$$
\begin{aligned}
E\left|I_{1}^{n}\right| & \leq c E\left(\sum_{t_{i} \in D_{n}}\left(N_{t_{i+1}}-N_{t_{i}}\right)^{2}\left(\int_{0}^{t_{i}}\left(G\left(t_{i+1}, s\right)-G\left(t_{i}, s\right)\right) d L_{s}\right)^{2}\right)^{1 / 2} \\
& \leq c E\left(\max _{t_{i} \in D_{n}}\left|N_{t_{i+1}}-N_{t_{i}}\right| \sum_{t_{i} \in D_{n}}\left(\int_{0}^{t_{i}}\left(G\left(t_{i+1}, s\right)-G\left(t_{i}, s\right)\right) d L_{s}\right)^{2}\right)^{1 / 2} \\
& \left.\leq c\left(E \max _{t_{i} \in D_{n}}\left|N_{t_{i+1}}-N_{t_{i}}\right|^{2}\right)\right)^{1 / 2}\left(E \sum_{t_{i} \in D_{n}}\left(\int_{0}^{t_{i}}\left(G\left(t_{i+1}, s\right)-G\left(t_{i}, s\right)\right) d L_{s}\right)^{2}\right)^{1 / 2} .
\end{aligned}
$$

Because of the continuity of $N$,

$$
E\left[\max _{t_{i} \in D_{n}}\left|N_{t_{i+1}}-N_{t_{i}}\right|^{2}\right] \rightarrow 0
$$

the second term is estimated as before by

$$
\left(E \int_{0}^{T} V_{2}^{2}(G)((s, T], s) d[L, L]_{s}\right)^{1 / 2}
$$

which is finite.

Now, by Schwarz's inequality

$$
\begin{aligned}
E\left|I_{2}^{n}\right| & \leq\left(E \sum_{t_{i} \in D_{n}}\left(N_{t_{i+1}}-N_{t_{i}}\right)^{2}\right)^{1 / 2}\left(E \sum_{t_{i} \in D_{n}}\left(\int_{t_{i}}^{t_{i+1}}\left(G\left(t_{i+1}, s\right)-G(s, s)\right) d L_{s}\right)^{2}\right)^{1 / 2} \\
& \leq\left(E\left([N, N]_{T}\right)\right)^{1 / 2}\left(E \int_{0}^{T}\left(G^{n}(s)-G(s, s)\right)^{2} d[L, L]_{s}\right)^{1 / 2}
\end{aligned}
$$

where $G^{n}$ is defined as above.

Since $E \int_{0}^{T}\left(G^{n}(s)-G(s, s)\right)^{2} d[L, L]_{s} \rightarrow 0$, we conclude that for every $t$

$$
\sum_{t_{i} \in D_{n}, t_{i} \leq t}\left(N_{t_{i+1}}-N_{t_{i}}\right)\left(A_{t_{i+1}}-A_{t_{i}}\right) \rightarrow 0
$$

in $\mathbf{L}^{1}$, and the covariation process $[N, A]$ is null for every continuous martingale $N$.

The proof of Theorem 4.1 is complete.

Unhappily we are not able to prove that $X$ admits a modification with càdlàg trajectories. However, in this direction, we have the following lemma.

Lemma 4.3: $A$ is continuous (hence $X$ is càdlàg) if the following additional condition is filled: 
$\left(H_{c}\right)$ : There exist $\delta>0, p>1$ and a function $a(u)$ meeting

$$
E\left[\left(\int_{0}^{T} a(u) d[L, L]_{u}\right)^{p}\right]<\infty
$$

such that for every $s, t, u$ holds

$$
(G(t, u)-G(s, u))^{2} \leq a(u)|t-s|^{\frac{1}{p}+\delta} .
$$

Proof : Let us take $s, t$ such that $0 \leq s \leq t \leq T$. We have with a constant $c$ changing from line to line:

$$
\begin{aligned}
E\left(A_{t}-A_{s}\right)^{2 p} \leq & E\left(\int_{0}^{t}(G(t, u)-G(u, u)) d L_{u}-\int_{0}^{s}(G(s, u)-G(u, u)) d L_{u}\right)^{2 p} \\
\leq & c E\left(\int_{0}^{s}(G(t, u)-G(s, u))^{2} d[L, L]_{u}\right)^{p} \\
& \quad+E\left(\int_{s}^{t}\left(G(t, u)-G(u, u)^{2} d[L, L]_{u}\right)^{p}\right. \\
\leq & c(t-s)^{1+p \delta} E\left(\int_{0}^{t} a(u) d[L, L]_{u}\right)^{p} \\
\leq & c(t-s)^{1+p \delta .}
\end{aligned}
$$

Hence we get the continuity of $A$ by Kolmogorov's Lemma.

An analogous result under Holder condition was already given in the paper ([1] ), Lemmas 2C and 2D.

We shall suppose by now that the processes given by (12) have a. s. càdlàg trajectories.

We are now interested in investigating conditions on $G$ in order to make $X$ a Dirichlet process or a weak Dirichlet process admitting a quadratic variation. For that let us consider the following hypotheses:

$\left(H_{4}\right)$ : For all $s, t \rightarrow G(t, s)$ has a bounded variation on $(s, \tau]$, for every $\tau \leq T$.

(We denote this variation $|G|((s, \tau], s)<\infty)$.

$\left(H_{5}\right): \quad E \int_{0}^{T}|G|((s, T], s) d[L, L]_{s}<\infty$

$\left(H_{6}\right)$ : For all $u, v, t \rightarrow G(t, u)$ and $t \rightarrow G(t, v)$ have a finite mutual quadratic covariation with the property $(S)$ on $(\max (u, v), T]$. 
(We denote this covariation $\left.[G(., u), G(., v)]_{\tau}\right)$. Moreover we suppose that the convergence involved to define the covariation, is uniform in $u, v$.

Of course $\left(H_{6}\right)$ implies that $t \rightarrow G(t, s)$ admits a quadratic variation on $(s, T]$ for all $s$ with the property $(S)$ and that $\left(H_{1}\right)$ is satisfied.

Theorem 4.2 (i) Let us assume $\left(H_{0}\right),\left(H_{3}\right),\left(H_{4}\right),\left(H_{5}\right)$. Then $X$ is a Dirichlet process. (i.e. $A$ is continuous and $[A, A] \equiv 0)$.

(ii) Let us assume $\left(H_{0}\right),\left(H_{2}\right),\left(H_{3}\right),\left(H_{6}\right)$. Then $X$ is a weak Dirichlet process. Moreover if we assume that the process $B$ defined by

$$
B_{t}=\int_{0}^{t}[G(., s), G(., s)]_{t} d[L, L]_{s}+2 \int_{0}^{t}\left(\int_{0}^{v}[G(., u), G(., v)]_{t} d L_{u}\right) d L_{v}
$$

is a càdlàg process, then $X$ and $A$ admit a quadratic variation such that: $[A, A]_{t}=B_{t}$ and

$$
[X, X]=[A, A]+[M, M] .
$$

Remark 4.2 (i) If $t \rightarrow G(t, s)$ is $C^{1}$ for every $s$, denoting $G_{1}(t, s)$ its derivative and assuming that $(t, s) \rightarrow G_{1}(t, s)$ is continuous on $[0,1]^{2}$, we get $A_{t}=\int_{0}^{t}\left(\int_{0}^{u} G_{1}(u, s) d L_{s}\right) d u$ by applying Fubini's theorem for stochastic integrals, so $\mathrm{X}$ is a semimartingale. This result is due to Protter ([13]).

(ii) In case of continuous martingale $L$, part 2) is due to Errami and Russo $([5])$ and $([6])$.

Proof: (i) First of all, we notice that our hypotheses imply that $\left(H_{1}\right)$ and $\left(H_{2}\right)$ are satisfied for any sequence $\left(D_{n}\right)$ of subdivisions with mesh tending to 0 . Since we can write

$A_{t_{i+1}}-A_{t_{i}}=\int_{0}^{t_{i}}\left(G\left(t_{i+1}, s\right)-G\left(t_{i}, s\right)\right) d L_{s}+\int_{t_{i}}^{t_{i+1}}\left(G\left(t_{i+1}, s\right)-G(s, s)\right) d L_{s}$,

for every $\varepsilon>0$ we get:

$$
E \sum_{t_{i} \in D_{n}}\left(A_{t_{i+1}}-A_{t_{i}}\right)^{2}
$$




$$
\begin{aligned}
\leq & E \sum_{t_{i} \in D_{n}} \int_{0}^{t_{i}}\left(G\left(t_{i+1}, s\right)-G\left(t_{i}, s\right)\right)^{2} d[L, L]_{s} \\
& +\sum_{t_{i} \in D_{n}} \int_{t_{i}}^{t_{i+1}}\left(G\left(t_{i+1}, s\right)-G(s, s)\right)^{2} d[L, L]_{s} \\
\leq & \max _{t_{i} \in D_{n}, \varepsilon \leq s \leq T}\left|G\left(t_{i+1}, s\right)-G\left(t_{i}, s\right)\right| E \sum_{t_{i} \in D_{n}} \int_{\varepsilon}^{t_{i}}\left|G\left(t_{i+1}, s\right)-G\left(t_{i}, s\right)\right| d[L, L]_{s} \\
& +E \sum_{t_{i} \in D_{n}} \int_{0}^{t_{i} \wedge \varepsilon}\left(G\left(t_{i+1}, s\right)-G\left(t_{i}, s\right)\right)^{2} d[L, L]_{s} \\
& +E \int_{0}^{T}\left(G^{n}(s)-G(s, s)\right)^{2} d[L, L]_{s} \\
\leq & \max _{t_{i} \in D_{n}, \varepsilon \leq s \leq T}\left|G\left(t_{i+1}, s\right)-G\left(t_{i}, s\right)\right| E \int_{\varepsilon}^{1}|G|((s, 1], s) d[L, L]_{s} \\
& +E \int_{0}^{\varepsilon} V_{2}^{2}(G)((s, T], s) d[L, L]_{s}+E \int_{0}^{T}\left(G^{n}(s)-G(s, s)\right)^{2} d[L, L]_{s}
\end{aligned}
$$

Using the following properties:

$$
\begin{aligned}
& \max _{t_{i} \in D_{n}, \varepsilon \leq s \leq T}\left|G\left(t_{i+1}, s\right)-G\left(t_{i}, s\right)\right| \rightarrow 0, \quad \text { when } n \rightarrow \infty, \\
& E \int_{\varepsilon}^{T}|G|((s, T], s) d[L, L]_{s}<\infty, \\
& E \int_{0}^{\varepsilon} V_{2}^{2}(G)((s, T], s) d[L, L]_{s} \rightarrow 0, \quad \text { when } \varepsilon \rightarrow, 0
\end{aligned}
$$

and

$$
E \int_{0}^{T}\left(G^{n}(s)-G(s, s)\right)^{2} d[L, L]_{s} \rightarrow 0, \quad \text { when } n \rightarrow \infty,
$$

we deduce that $[A, A] \equiv 0$. By the inequality

$$
\max _{t_{i} \in D_{n}}\left|A_{t_{i+1}}-A_{t_{i}}\right|^{2} \leq \sum_{t_{i} \in D_{n}}\left(A_{t_{i+1}}-A_{t_{i}}\right)^{2},
$$

we get that $A$ is continuous.

(ii) Taking into account Proposition 2.1 we have only to prove the property $S$ for the process defined by the right hand side of formula (13). Let us notice first that formula (13) is well-defined, as we take as integrant of $d L_{s}$ for the last term the predictable projection of the optional process

$$
\left(\int_{0}^{s}[G(., u), G(., s)]_{t} d L_{u}\right)_{s \leq t} .
$$


See for example Dellacherie -Meyer ([4]), Chap.VI for details.

Taking into account that

$E \int_{0}^{T}\left(G^{n}(s)-G(s, s)\right)^{2} d[L, L]_{s} \rightarrow 0$ when $n \rightarrow \infty$,

we have:

$$
\begin{aligned}
& {[A, A]_{t}=\lim _{n} \sum_{t_{i} \in D_{n}, t_{i} \leq t}\left(A_{t_{i+1}}-A_{t_{i}}\right)^{2}} \\
& =\lim _{n} \sum_{t_{i} \in D_{n}, t_{i} \leq t}\left(\int_{0}^{t_{i}}\left(G\left(t_{i+1}, s\right)-G\left(t_{i}, s\right)\right) d L_{s}\right)^{2} \\
& =\lim _{n} 2 \sum_{t_{i} \in D_{n}, t_{i} \leq t} \int_{0}^{t_{i}}\left(\int_{0}^{s}\left(G\left(t_{i+1}, u\right)-G\left(t_{i}, u\right)\right) d L_{u}\right)_{-}\left(G\left(t_{i+1}, s\right)-G\left(t_{i}, s\right)\right) d L_{s} \\
& \quad \quad+\lim _{n} \sum_{t_{i} \in D_{n}, t_{i} \leq t} \int_{0}^{t_{i}}\left(G\left(t_{i+1}, s\right)-G\left(t_{i}, s\right)\right)^{2} d[L, L]_{s} \\
& =2 \lim _{n} I_{1}^{n}(t)+\lim _{n} I_{2}^{n}(t) .
\end{aligned}
$$

Then

$$
I_{2}^{n}(t)=\sum_{k} \int_{t_{k-1}}^{t_{k}} \sum_{i>k, t_{i} \leq t}\left(G\left(t_{i+1}, s\right)-G\left(t_{i}, s\right)\right)^{2} d[L, L]_{s}
$$
gence.

This sequence converges to $\int_{0}^{t}[G(., s)]_{t} d[L, L]_{s}$ by dominated conver-

On the other hand, $I_{1}^{n}(t)$ can be written

$$
\begin{aligned}
I_{1}^{n}(t) & =\sum_{k} \int_{t_{k-1}}^{t_{k}}\left(\int_{0}^{s} \sum_{i>k, t_{i} \leq t}\left(G\left(t_{i+1}, u\right)-G\left(t_{i}, u\right)\right)\left(G\left(t_{i+1}, s\right)-G\left(t_{i}, s\right)\right) d L_{u}\right)_{-} d L_{s} \\
& =\int_{0}^{\rho^{n}(t)}\left(\int_{0}^{s}\left[G^{n}(., u), G^{n}(., s)\right]_{t} d L_{u}\right)_{-} d L_{s} .
\end{aligned}
$$

For any optional process $Y$, let us write $Y^{P}$ its predictable projection. Then noticing that

$$
\left(\int_{0}^{s}\left[G^{n}(., u), G^{n}(., s)\right]_{t} d L_{u}\right)_{-}=\left(\int_{0}^{s}\left[G^{n}(., u), G^{n}(., s)\right]_{t} d L_{u}\right)^{P}
$$

and from classical properties of predictable projections, we get for $t \in D_{n}$

$$
E\left(I_{1}^{n}(t)-\int_{0}^{t}\left(\int_{0}^{s}[G(., u), G(., s)]_{t} d L_{u}\right) d L_{s}\right)^{2}
$$




$$
\begin{aligned}
& =E\left[\int_{0}^{t}\left[\left(\int_{0}^{s}\left(\left[G^{n}(., u), G^{n}(., s)\right]_{t}-[G(., u), G(., s)]_{t}\right) d L_{u}\right)^{P}\right]^{2} d<L, L>_{s}\right] \\
& \leq E\left[\int_{0}^{t}\left[\left(\int_{0}^{s}\left(\left[G^{n}(., u), G^{n}(., s)\right]_{t}-[G(., u), G(., s)]_{t}\right) d L_{u}\right)^{2}\right]^{P} d<L, L>_{s}\right] \\
& =E\left[\int_{0}^{t}\left(\int_{0}^{s}\left(\left[G^{n}(., u), G^{n}(., s)\right]_{t}-[G(., u), G(., s)]_{t}\right) d L_{u}\right)^{2} d<L, L>_{s}\right]
\end{aligned}
$$

We show now that for any $t \in \bigcup_{n} D_{n}$

$\int_{0}^{t}\left(\int_{0}^{s}\left(\left[G^{n}(., u), G^{n}(., s)\right]_{t}-[G(., u), G(., s)]_{t}\right) d L_{u}\right)^{2} d<L>_{s} \stackrel{\mathbf{P}}{\longrightarrow} 0, \quad$ when $n \rightarrow \infty$.

Note that

$$
\begin{aligned}
& E\left(\int_{0}^{s}\left(\left[G^{n}(., u), G^{n}(., s)\right]_{t}-[G(., u), G(., s)]_{t}\right) d L_{u}\right)^{2} \\
& \quad=E\left(\int_{0}^{s}\left(\left[G^{n}(., u), G^{n}(., s)\right]_{t}-[G(., u), G(., s)]_{t}\right)^{2} d<L, L>_{u}\right) .
\end{aligned}
$$

But for every $u, s, t,\left[G^{n}(., u), G^{n}(., s)\right]_{t}-[G(., u), G(., s)]_{t} \rightarrow 0$ when $n \rightarrow \infty$, and we have the estimation

$$
\begin{aligned}
& \left|\left[G^{n}(., u), G^{n}(., s)\right]_{t}-[G(., u), G(., s)]_{t}\right| \\
& \left.\quad \leq 1 / 2\left(\left[G^{n}(., u)\right]_{t}+\left[G^{n}(., s)\right]_{t}+[G(., u)]_{t}+G(., s)\right]_{t}\right) .
\end{aligned}
$$

¿From $\left(H_{6}\right)$ this last term is bounded, hence by dominated convergence

$$
E\left(\int_{0}^{s}\left(\left[G^{n}(., u), G^{n}(., s)\right]_{t}-[G(., u), G(., s)]_{t}\right) d L_{u}\right)^{2} \rightarrow 0
$$

for every $s, t$. So, we can get easily (18) by localisation of $L$.

We are finished as soon as we remark that using the continuity of process $<L, L>$, for every $t$

$$
\begin{aligned}
E\left(I_{1}^{n}(t)-\right. & \left.I_{1}^{n}\left(\rho^{n}(t)\right)-\int_{\rho^{n}(t)}^{t}\left(\int_{0}^{s}[G(., u), G(., s)] d L_{u}\right) d L_{s}\right)^{2} \\
& =E\left(\int_{\rho^{n}(t)}^{t}\left(\int_{0}^{s}[G(., u), G(., s)] d L_{u}\right) d L_{s}\right)^{2} \\
& =E \int_{\rho^{n}(t)}^{t}\left(\int_{0}^{s}[G(., u), G(., s)] d L_{u}\right)^{2} d<L, L>_{s}
\end{aligned}
$$

converges to 0 when $n \rightarrow \infty$.

Example 1: Fractional normal processes of index $H>1 / 2$ 
We consider the case where $L$ is a normal martingale (i.e. a square integrable martingale with predictable quadratic variation $\left\langle L, L>_{t}=t\right.$ ), and $G(t, s)$ given for $t \geq s$ by:

$$
G(t, s)=c s^{1 / 2-H} \int_{s}^{t} u^{H-1 / 2}(u-s)^{H-3 / 2} d u
$$

with $c$ constant. Of course, when $L$ is a standard Brownian motion, $X$ is the classical fractional Brownian motion.

Let us check that $\left(H_{3}\right),\left(H_{4}\right)$ and $\left(H_{5}\right)$ are satisfied.

$$
\begin{aligned}
|G|((s, T], s) & =c s^{1 / 2-H} \int_{s}^{T} u^{H-1 / 2}(u-s)^{H-3 / 2} d u \\
& \leq c s^{1 / 2-H} \int_{s}^{T}(u-s)^{H-3 / 2} d u \\
& =c s^{1 / 2-H} \frac{1}{H-1 / 2}(1-s)^{H-1 / 2} d u \\
& \leq \frac{c}{H-1 / 2} s^{1 / 2-H}
\end{aligned}
$$

and

$$
\int_{0}^{T}|G|((s, T], s) d s \leq \frac{c}{(H-1 / 2)(3 / 2-H)}
$$

hence finally

$$
\int_{0}^{T}\left|G^{2}\right|((s, T], s) d s \leq \frac{c}{(H-1 / 2)(2-2 H)} .
$$

This means that, for every normal martingale $L$, the process $X$ defined by $X_{t}=\int_{0}^{t} G(t, s) d L_{s}$ is a Dirichlet process. Since $G(s, s)=0$, its martingale part is null. In particular $X$ is continuous, even if $L$ is not.

Example 2: Weak Dirichlet process driven by a Brownian motion

For this example we take $L=B$ a standard Brownian motion and $G(t, s)=\beta(t) f(s)$ for $t \geq s$, where $t \rightarrow \beta(t)$ is a fixed Brownian trajectory such that its quadratic variation is $[\beta(.)]_{t}=t$, and $f$ is a real continuous function on $[0, T]$.

Here we can apply part 2) of Theorem 4.2. Actually:

$$
[G(;, u), G(., v)]_{t}=\lim _{t_{i+1} \leq t, t_{i} \geq \max \{u, v\}} \sum_{i}\left(\beta\left(t_{i+1}\right)-\beta\left(t_{i}\right)\right)^{2} f(u) f(v)
$$


and this term converges uniformly in $u, v$ to $(t-\max \{u, v\}) f(u) f(v)$.

Therefore we get the decomposition $X=M+A$, with

$$
M_{t}=\int_{0}^{t} \beta(s) f(s) d B_{s}
$$

and the formula (13) gives the quadratic variation of $A$

$$
[A, A]_{t}=\int_{0}^{t}(t-s) f^{2}(s) d s+2 \int_{0}^{t} \int_{0}^{s}(t-s) f(u) f(s) d B_{u} d B_{s}
$$

which can be written

$$
[A, A]_{t}=\int_{0}^{t}\left(\int_{0}^{s} f(u) d B_{u}\right)^{2} d s .
$$

In particular the process $\left(\int_{0}^{t} \int_{0}^{s}(t-s) f(u) f(s) d B_{u} d B_{s}\right)_{t}$ has a finite variation.

Since $E\left([A, A]_{t}\right)=\int_{0}^{t}(t-s) f^{2}(s) d s \neq 0, X$ is not a Dirichlet process; however, Theorems 4.1 and 4.2 ensure that $X$ is a weak Dirichlet process admitting quadratic variation.

Actually, this example 2 is a particular case of the following:

\section{Example 3}

Let us consider $G(t, s)$ under the form

$$
G(t, s)=\int_{s}^{t} f(u, s) d \beta_{u}
$$

where for every $s<t, u \rightarrow f(u, s)$ has a bounded variation on $(s, t]$, and $(u, s) \rightarrow f(u, s)$ is continuous on $\{(u, s): 0 \leq u \leq s \leq T\}$. We denote $d f_{u}(u, s)$ the measure associated to the variation process. We assume also that $\beta$ is a deterministic real continuous on $[0, T]$ function admitting a quadratic variation along $\left(D_{n}\right)$, and $\beta_{0}=0$.

$X_{t}=A_{t}$ and we shall prove the Fubini type formula :

$$
A_{t}=\int_{0}^{t}\left(\int_{s}^{t} f(u, s) d \beta_{u}\right) d L_{s}=\int_{0}^{t}\left(\int_{0}^{u} f(u, s) d L_{s}\right) d \beta_{u} .
$$

Indeed, $A$ admits a quadratic variation along $\left(D_{n}\right)$ given by

$$
[A, A]_{t}=\int_{0}^{t}\left(\int_{0}^{s} f(u, s) d L_{u}\right)^{2} d[\beta, \beta]_{s} .
$$


Actually, taking into account that $[\beta, f(., s)]=0$, we get

$$
\int_{s}^{t} f(u, s) d \beta_{u}=\beta_{t} f(t, s)-\beta_{s} f(s, s)-\int_{s}^{t} \beta_{u} d f_{u}(u, s) .
$$

Then

$$
A_{t}=\beta_{t} \int_{0}^{t} f(t, s) d L_{s}-\int_{0}^{t} \beta_{s} f(s, s) d L_{s}-\int_{0}^{t}\left(\int_{s}^{t} \beta_{u} d f_{u}(u, s)\right) d L_{s} .
$$

¿From Theorem 4.2 (i) the process $Y$ defined by $Y_{t}=\int_{0}^{t} f(t, s) d L_{s}$ is a Dirichlet process and $[\beta, Y]=0$, then by integration by parts, we get;

$$
\beta_{t} Y_{t}=\int_{0}^{t} Y_{s} d \beta_{s}+\int_{0}^{t} \beta_{s} d Y_{s}
$$

And by using the sequence $\left(D_{n}\right)$

$$
\begin{gathered}
\int_{0}^{t} \beta_{s} d Y s=\lim _{D_{n}} \sum_{t_{i} \in D_{n}, t_{i} \leq t} \beta_{t_{i}}\left(Y_{t_{i+1}}-Y_{t_{i}}\right) \\
=\lim _{D_{n}} \sum_{t_{i} \in D_{n}, t_{i} \leq t} \beta_{t_{i}} \int_{t_{i}}^{t_{i+1}} f\left(t_{i+1}, s\right) d L_{s} \\
+\lim _{D_{n}} \sum_{t_{i} \in D_{n}, t_{i} \leq t} \beta_{t_{i}} \int_{0}^{t_{i}}\left(f\left(t_{i+1}, s\right)-f\left(t_{i}, s\right)\right) d L_{s} \\
=\int_{0}^{t} \beta_{s} f(s, s) d L_{s}+\int_{0}^{t}\left(\int_{s}^{t} \beta_{u} d f_{u}(u, s)\right) d L_{s},
\end{gathered}
$$

then we deduce the formula (18).

\section{Appendix}

\subsection{A process with finite energy but without quadratic vari- ation along the dyadics.}

Let $D_{k}$ be the $k$-th dyadic subdivision of $[0,1]$, that is $t_{j}^{k}=\frac{j}{2^{k}}, 0 \leq j \leq 2^{k}$.

We are willing to build a deterministic function $x$ such that $S_{k}=1$ if $k$ is even and greater than 2 , and $S_{k}=2$ for $k$ odd. Such a funcion has obviously finite energy along the sequence $\left(D_{k}\right)_{k}$ (and indeed its energy is equal to 2 , although is would be equal to 1 along the sequence $\left.\left(D_{2 k}\right)_{k}\right)$, but has no quadratic variation since the sequence $\left(S_{k}\right)$ has 2 accumulation points.

Let us begin with defining $x_{0}=x_{1}=0$ and $x_{1 / 2}=1$, so that $S_{1}=2$. 
At the second step, we define $x_{1 / 4}=x_{3 / 4}=1 / 2$, so that $S_{2}=1$

In order to make our construction clear, we go into details for the third step.

We want to define $x_{j / 8}$ for odd $j$ in order that $S_{3}=2$. The idea is to compute $x_{j / 8}$ such that

$$
\left(x_{\frac{j}{8}}-x_{\frac{j-1}{8}}\right)^{2}+\left(x_{\frac{j+1}{8}}-x_{\frac{j}{8}}\right)^{2}=2 \times\left(x_{\frac{j+1}{8}}-x_{\frac{j-1}{8}}\right)^{2} .
$$

Actually, this amounts to find a solution $y$ to an equation like

$$
(a-y)^{2}+(b-y)^{2}=2 \times(a-b)^{2} .
$$

As equation (19) has two solutions, namely $((1+\sqrt{3}) a+(1-\sqrt{3}) b) / 2$ and $((1-\sqrt{3}) a+(1+\sqrt{3}) b) / 2$, we have 2 possible choices for each $x_{j / 8}$ with odd $j$ in order that $S_{3}=2$.

This process is then iterated as follows :

1. Assume that we have constructed $x_{\frac{j}{2^{2 k-1}}}, 0 \leq j \leq 2^{2 k-1}$, such that $S_{2 k-1}=2$ for some $k$. Then we put $x_{\frac{2 j+1}{2^{2 k}}}=\left(x_{\frac{2 j}{2^{2 k}}}+x_{\frac{2 j+2}{2^{2 k}}}\right) / 2$ (so that it is the middle of its neighbours). Then it is readily checked that $S_{2 k}=1$.

2. Now we have to choose the $x_{\frac{2 j+1}{2^{2 k+1}}}$ 's. We will proceed as was done above for $k=1$. Namely, we can always choose $y=x_{\frac{2 j+1}{2^{2 k+1}}}$ so that it solves equation (19) with $a=x_{\frac{2 j}{2^{2 k+1}}}$ and $b=x_{\frac{2 j+2}{2^{2 k+1}}}$, and the result follows the same lines as for $k=1$.

It remains to check that we can build a real continuous function $x$ on $[0,1]$ with the specified values on the dyadics.

Let $x^{n}$ be the piecewise linear function joining the points constructed at rank $n$. We will show that the sequence $\left(x^{n}\right)$ satisfies a uniform Cauchy criterion, which will give the claim.

First note that it is obvious (again from the solution of equation (19) that any two neighbours at rank $2 k$ or $2 k+1$ are far from each other at most $\left((1+\sqrt{3} / 4)^{k}\right.$. In other words, we have always

$$
\left|x_{\frac{i+1}{2^{n}}}^{n}-x_{\frac{i}{2^{n}}}^{n}\right| \leq\left(\frac{1+\sqrt{3}}{4}\right)^{\frac{n}{2}} .
$$

Now, fix $\varepsilon>0$. For positive $n$ and $p$ and for $t \in[0,1]$, let $t_{i}^{n}$ be the closest to $t$ point in $D_{n}$, and $t_{j}^{n+p}$ the closest to $t$ point in $D_{n+p}$. Without loss of generality we will assume that $t_{i}^{n} \leq t_{j}^{n+p} \leq t_{i+1}^{n}$. We have then 


$$
\begin{array}{cc}
\left|x_{t}^{n}-x_{t}^{n+p}\right| & \leq\left|x_{t}^{n}-x_{t_{i}^{n}}^{n}\right|+\left|x_{t_{i}^{n}}^{n}-x_{t_{i}^{n}}^{n+p}\right| \\
& +\left|x_{t_{i}^{n}}^{n+p}-x_{t_{j}^{n+p}}^{n+p}\right|+\left|x_{t_{j}^{n+p}}^{n+p}-x_{t}^{n+p}\right| .
\end{array}
$$

¿From (20) and the definition of $x^{n}$ it is obvious that the first and last terms in the right-hand side of (21) can be made as small as wanted (say less than $\varepsilon / 3$ ), uniformly in $t$ and $p$, for $n$ large enough. Moreover, as $D_{n} \subset D_{n+p}$, the second term is identically zero.

Hence it remains to uniformly estimate $\left|x_{t_{i}^{n}}^{n+p}-x_{t_{j}^{n+p}}^{n+p}\right|$ (note that in principle, at most $2^{p}$ points of $D_{n+p}$ may lay between $t_{i}^{n}$ and $t_{j}^{n+p}$, so that there is no trivial uniform in $p$ majorization of such a sequence).

Let us put $t_{n}^{i}=k / 2^{n}$

We assume for instance that $l:=x_{k / 2^{n}} \leq h: x_{(k+1) / 2^{n}}$ and that $n$ is odd. Then clearly $l \leq x_{(2 k+1) / 2^{n+1}} \leq h\left(\right.$ since $\left.x_{(2 k+1) / 2^{n+1}}=(l+h) / 2\right)$, and if we choose $x_{(4 k+1) / 2^{n+2}}=((1+\sqrt{3}) l+(1-\sqrt{3})(l+h) / 2) / 2$ and $x_{(4 k+3) / 2^{n+2}}=((1+\sqrt{3})(h+l) / 2+(1-\sqrt{3}) h) / 2$, we can see that both values lay in $[l-(\sqrt{3}-1)(h-l) / 4, h]$. Keeping $(20)$ in mind, we conclude that for every point $s$ in $D_{n+2} \cap\left[k / 2^{n},(k+1) / 2^{n}\right]$, and for every $p \geq 2$,

$$
l-(\sqrt{3}-1) / 4\left(\frac{1+\sqrt{3}}{4}\right)^{\frac{n}{2}} \leq x_{s}^{n+p} \leq h .
$$

If we iterate this procedure, it is straightforward now that for every point $s$ in $D_{n+2 m} \cap\left[k / 2^{n},(k+1) / 2^{n}\right]$, and for every $p \geq 2 m$,

$$
l-\frac{\sqrt{3}-1}{4}\left(\frac{1+\sqrt{3}}{4}\right)^{\frac{n}{2}} \sum_{i=0}^{m-1}\left(\frac{1+\sqrt{3}}{4}\right)^{i} \leq x_{s}^{n+p} \leq h
$$

and eventually, for every point $s$ in $\bigcup_{m} D_{n+2 m} \cap\left[k / 2^{n},(k+1) / 2^{n}\right]$, and for every $p \geq 0$

$$
l-\frac{\sqrt{3}-1}{4}\left(\frac{1+\sqrt{3}}{4}\right)^{\frac{n}{2}} \frac{4}{3-\sqrt{3}} \leq x_{s}^{n+p} \leq h .
$$

At last, il follows that the third term in the right-hand of (21) can be made as small as wanted, say less than $\varepsilon / 3$, for $n$ big enough,uniformly in $t$ and $p$, and finally we checked the uniform Cauchy criterion for the sequence of functions $\left(x^{n}\right)$. Hence this sequence converges to a continuous function $x$, such that by construction, for every $k$ 


$$
\sum_{i \in D_{2 k}}\left(x_{i+1}-x_{i}\right)^{2}=1
$$

and

$$
\sum_{i \in D_{2 k+1}}\left(x_{i+1}-x_{i}\right)^{2}=2 .
$$

This function has finite energy, but no quadratic variation along the dyadics.

\subsection{A continuous function with discontinuous pre-quadratic variation}

We consider the function introduced in [3], Example 1, that is the piecewise affine function $X$ such that $X_{t}=0$ at each $t=1-2^{1-2 p}, X_{t}=1 / p^{1 / 2}$ for $t=1-2^{-2 p}$, and $X$ is affine between these points. If we define moreover $X_{1}=0, X$ is a continuous fonction on $[0,1]$.

It is clear that $X$ is a fonction of finite variation, hence of zero quadratic variation on every $[0, t]$ with $t<1$.

On the other hand, it was proven in [3] that $X$ has an infinite quadratic variation on $[0,1]$ along $S:=\left\{1-2^{-2 k}, k \geq 1\right\}$.

For $n>0$, we define now a subdivision $\pi_{n}$ of $[0,1]$ as follows :

$$
\pi_{n}=\bigcup_{j \leq 2^{2 n}-1}\left\{j 2^{-2 n}\right\} \cup \bigcup_{k \geq n}\left\{1-2^{-2 k}\right\} .
$$

It is straightforward that for every $n X$ has an infinite quadratic variation along $\pi_{n}$, although its quadratic variation along $\pi_{n} \cap[0, t]$ goes to zero as $n \rightarrow \infty$ for every $t<1$.

Note that if we modify our example in order that $X_{t}=1 / p$ for $t=$ $1-2^{-2 p}, p>0$, everything else remaining unchanged, the pre-quadratic variation of $X$ on $[0, t]$ is equal to zero if $t<1$, but finite and non zero for $t=1$.

\subsection{A continuous in probability process may admit no càdlàg modification.}

Let $X$ be the piecewise affine function such that $X_{t}=0$ at each $t=1-2^{1-2 p}$, $X_{t}=1$ for $t=1-2^{-2 p}$, and $X$ is affine between these points. If we define 
moreover $X_{t}=0$ outside $[0,1)$, we get a discontinuity of the second kind at 1 .

Now define the process $Y$ as follows : $Y_{t}=X_{t-T} \mathbf{1}_{t>T}$, where $T$ is a random variable uniformly distributed on $[0,1]$, then $Y$ is continuous in probability, but every path of $Y$ has almost surely a discontinuity of the second kind between times 1 and 2 .

Note that this result remains true even if we ask our process to have a quadratic variation along a sequence $\left(\pi_{n}\right)$.

\section{References}

[1] M.A. Berger, V.J. Mizel, Volterra Equations with Itô integrals-I, J. Integ. Eq. 2 (1980), 187-245.

[2] F. Coquet, J. Mémin and L. Słomiński, On Non-Continuous Dirichlet Processes, J. Theor. Probab., 16 (2003), 197-216.

[3] F. Coquet and L. Słomiński, On the convergence of Dirichlet Processes, Bernoulli, 5 (1999), 615-639.

[4] C. Dellacherie, P.A. Meyer, Probabilités et potentiel, Chap. V à VIII, 1980, Hermann, Paris.

[5] M. Errami, F. Russo, n-covariation, generalized Dirichlet processes and calculus with respect to finite cubic variation processes, Stochastic process. appl., 104, (2003), 259-299.

[6] M. Errami, F. Russo, Covariation de convolution de martingales, $C$. R. Acad. Sci. Paris, t. 326, Série I,p. 601-606, 1998.

[7] H. Föllmer, Calcul d'Ito sans Probabilités, in Séminaire de Probabilités XV, Lecture Notes in Maths 850, Springer-Verlag (1981), $143-150$.

[8] H. Föllmer, Dirichlet Processes, in Séminaire de Probabilités XV, Lecture Notes in Maths 851, Springer-Verlag (1981), 476-478.

[9] S.E. Graversen and M. Rao, Quadratic variation and Energy, Nagoya Math. J., 100 (1985), 163-180. 
[10] J. Jacod, Convergence en loi de semimartigales et variation quadratique, in Séminaire de Probabilités XV, Lecture Notes in Maths 850, Springer, Berlin, 1981.

[11] J. Jacod, A. Shiryaev, Limit Theorems for Stochastic Processes, Springer, Berlin 1987.

[12] M. Métivier, Notions fondamentales de la théorie des probabilités, 1968, Dunod, Paris.

[13] P. Protter, Volterra equations driven by semimartingales, The Annals of Probability, 1985, Vol. 13, No 2, 519-530.

[14] F. Russo, P. Vallois, The generalized covariation process and Ito formula, Stochastic process. appl, 59 (1995), 81-104. 